\title{
Inference for the Weibull Distribution: A tutorial
}

\author{
F. W. Scholz ${ }^{\mathrm{a}, \boldsymbol{U}}$

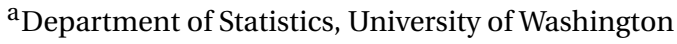

Abstract $\square$ This tutorial deals with the 2-parameter Weibull distribution. In particular it covers the construction of confidence bounds and intervals for various parameters of interest, the Weibull scale and shape parameters, its quantiles and tail probabilities. These bounds were pioniered in Thoman, Bain, and Antle, 1969, Thoman, Bain, and Antle, 1970, Bain, 1978, and Bain and Engelhardt, 1991, where tables for their computation were given. These tables were based on simulations and show occasional irregularities. In conjunction with this tutorial we provide R code to perform various tasks (generating plots, perform simulations). It greatly simplifies the application of these methods over trying to use the tables available so far. Today's computing availability and speed makes this very viable. For the freely available $\mathrm{R}$ computing platform we refer to R Core Team, 2015. The text identifies R code by using courier font in appropriate places.

Keywords - Weibull distribution; confidence intervals; inference

fscholz@u.washington.edu

\section{Introduction}

For parameters $\alpha>0$ and $\beta>0$ the 2-parameter Weibull cumulative distribution function (cdf) is defined as

$$
F_{\alpha, \beta}(x)= \begin{cases}1-\exp \left[-\left(\frac{x}{\alpha}\right)^{\beta}\right] & \text { for } x \geq 0 \\ 0 & \text { for } x<0\end{cases}
$$

We also write $X \sim \mathbb{W}(\alpha, \beta)$ when $X$ has this distribution function, i.e., $P(X \leq x)=F_{\alpha, \beta}(x)$. The parameters $\alpha$ and $\beta$ are referred to as scale and shape parameter, respectively. The Weibull density has the following form

$$
f_{\alpha, \beta}(x)=F_{\alpha, \beta}^{\prime}(x)=\frac{d}{d x} F_{\alpha, \beta}(x)=\frac{\beta}{\alpha}\left(\frac{x}{\alpha}\right)^{\beta-1} \exp \left[-\left(\frac{x}{\alpha}\right)^{\beta}\right] \text {. }
$$

For $\beta=1$ the Weibull distribution coincides with the exponential distribution with mean $\alpha$. In general, $\alpha$ represents the .632-quantile of the Weibull distribution regardless of the value of $\beta$ since $F_{\alpha, \beta}(\alpha)=1-\exp (-1) \approx .632$ for all $\beta>0$. Figure 1 (produced by densities ()) shows a representative collection of Weibull densities. Note that the spread of the Weibull distributions around $\alpha$ gets smaller as $\beta$ increases. The reason for this will become clearer later when we discuss the log-transform of Weibull random variables.

The $m^{\text {th }}$ moment of the Weibull distribution is

$$
\mathrm{E}\left(X^{m}\right)=\alpha^{m} \Gamma(1+m / \beta)
$$

and thus the mean and variance are given by

$$
\mu=E(X)=\alpha \Gamma(1+1 / \beta)
$$

and

$$
\sigma^{2}=\alpha^{2}\left[\Gamma(1+2 / \beta)-\{\Gamma(1+1 / \beta)\}^{2}\right]
$$

Its $p$-quantile, defined by $P\left(X \leq x_{p}\right)=p$, is

$$
x_{p}=\alpha(-\log (1-p))^{1 / \beta} \text {. }
$$

For $p=1-\exp (-1) \approx .632$ (i.e., $-\log (1-p)=1$ ) we have $x_{p}=\alpha$ regardless of $\beta$, as pointed out previously. For that reason one also calls $\alpha$ the characteristic life of the Weibull distribution. The term life comes from the common use of the Weibull distribution in modeling lifetime data. More on this later.

For parameters $\tau \in R, \alpha>0$ and $\beta>0$ the 3-parameter Weibull cdf is defined as

$$
F_{\alpha, \beta}(x)= \begin{cases}1-\exp \left[-\left(\frac{x-\tau}{\alpha}\right)^{\beta}\right] & \text { for } x \geq \tau \\ 0 & \text { for } x<\tau\end{cases}
$$

We will not deal with this more general form of the Weibull distribution. The method of maximum likelihood does not work well in this context.

\section{Minimum Closure and Weakest Link Property}

The Weibull distribution has the following minimum closure property: If $X_{1}, \ldots, X_{n}$ are independent and identically distributed (i.i.d.) with $X_{i} \sim \mathscr{W}\left(\alpha_{i}, \beta\right), i=1, \ldots, n$, then

$$
\begin{aligned}
P\left(\min \left(X_{1}, \ldots, X_{n}\right)>t\right) & =P\left(X_{1}>t, \ldots, X_{n}>t\right)=\prod_{i=1}^{n} P\left(X_{i}>t\right) \\
& =\prod_{i=1}^{n} \exp \left[-\left(\frac{t}{\alpha_{i}}\right)^{\beta}\right] \\
& =\exp \left[-t^{\beta} \sum_{i=1}^{n} \frac{1}{\alpha_{i}^{\beta}}\right]=\exp \left[-\left(\frac{t}{\alpha^{\star}}\right)^{\beta}\right]
\end{aligned}
$$

The Quantitative Methods for Psychology 
Figure 1 a A Collection of Weibull Densities with $\alpha=10000$ and Various Shapes

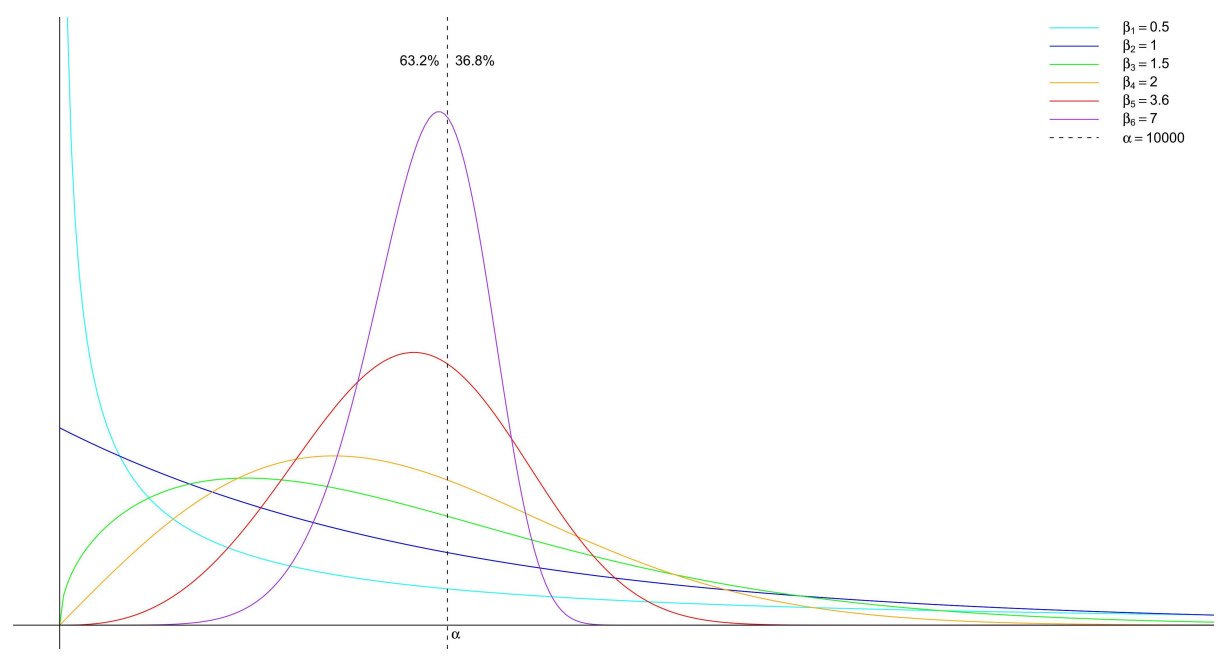

with

$$
\alpha^{\star}=\left(\sum_{i=1}^{n} \frac{1}{\alpha_{i}^{\beta}}\right)^{-1 / \beta},
$$

i.e., $\min \left(X_{1}, \ldots, X_{n}\right) \sim \mathbb{W}\left(\alpha^{\star}, \beta\right)$. This is reminiscent of the closure property for the normal distribution under summation, i.e., if $X_{1}, \ldots, X_{n}$ are independent with $X_{i} \sim \mathscr{N}\left(\mu_{i}, \sigma_{i}^{2}\right)$ then

$$
\sum_{i=1}^{n} X_{i} \sim \mathscr{N}\left(\sum_{i=1}^{n} \mu_{i}, \sum_{i=1}^{n} \sigma_{i}^{2}\right) .
$$

This summation closure property plays an essential role in proving the central limit theorem: Sums of independent random variables (not necessarily normally distributed) have an approximate normal distribution, subject to some mild conditions concerning the distribution of such random variables. There is a similar result from Extreme Value Theory, see Gumbel, 1958, Coles, 2001,Embrechts, Klüppelberg, and Mikosch, 1997, Castillo, 1988, that says: The minimum of $n$ independent, identically distributed random variables (not necessarily Weibull distributed, but subject to some mild conditions concerning the distribution of such random variables) has for large $n$ one of three possible approximate distributions: the above Weibull distribution, the Gumbel distribution (specified later), and the negative Weibull distribution (of little interest in reliability theory). This extreme value theory result is also referred to as the "weakest link" motivation for the Weibull distribution.

The Weibull distribution is appropriate when trying to characterize the random strength of materials or the random lifetime of some system. This is related to the weakest link property as follows. A piece of material can be viewed as a concatenation of many smaller material cells, each of which has its random breaking strength $X_{i}$ when subjected to tensile stress. Thus the strength of the concatenated total piece is the strength of its weakest link, namely $\min \left(X_{1}, \ldots, X_{n}\right)$, i.e., approximately Weibull.

Similarly, a system can be viewed as a collection of many parts or subsystems, each of which has a random lifetime $X_{i}$. If the system is defined to be in a failed state whenever any one of its parts or subsystems fails, then the system lifetime is $\min \left(X_{1}, \ldots, X_{n}\right)$, i.e., approximately Weibull.

Publications concerning the Weibull distribution, its theoretical properties and practical applications have seen a dramatic rise as is illustrated gaphically by Heller (1985) over the period 1939-1975. Googling "Weibull distribution" in 2008 produced 185,000 hits while "normal distribution" had 2,420,000 hits. In 2015 these counts had risen to 426,000 and $6,020,000$, respectively. Figure $2^{1}$ shows the "real thing," a reference to a remark by Weibull that he is of Hungarian origin and that the Hungarian "Valodi" translates to "real thing", see Heller (1985).

The Weibull distribution is very popular among engineers. One reason for this is that the Weibull cdf has a closed form which is not the case for the normal cdf $\Phi(x)$. However, in today's computing environment one could argue that point since typically the computation of even $\exp (x)$ requires computing. That this can be accomplished on most calculators is also moot since many calculators also give you $\Phi(x)$. For some limited period this popularity explanation may have been quite valid. Another reason for the popularity of the Weibull distribution among engineers

\footnotetext{
${ }^{1}$ Many thanks to Sam Saunders for this photo.
} 
may be that Weibull's most famous paper, Weibull, 1951, originally submitted to a statistics journal and rejected, was eventually published in an engineering journal.

Quoting Göran W. Weibull, 1981, http://www.garfield. library.upenn.edu/classics1981/A1981LD32400001.pdf : "... he tried to publish an article in a well-known British journal. At this time, the distribution function proposed by Gauss was dominating and was distinguishingly called the normal distribution. By some statisticians it was even believed to be the only possible one. The article was refused with the comment that it was interesting but of no practical importance. That was just the same article as the highly cited one published in 1951."

Saunders, 1975: 'Professor Wallodi (sic) Weibull recounted to me that the now famous paper of his "A Statistical Distribution of Wide Applicability", in which was first advocated the "Weibull" distribution with its failure rate a power of time, was rejected by the Journal of the American Statistical Association as being of no interest. Thus one of the most influential papers in statistics of that decade was published in the Journal of Applied Mechanics. See [35]. (Maybe that is the reason it was so influential!)'

\section{The Hazard Function}

The hazard function for any nonnegative random variable with cdf $F(x)$ and density $f(x)$ is defined as $h(x)=f(x) /(1-$ $F(x)$ ). It is usually employed for distributions that model random lifetimes and it relates to the probability that a lifetime comes to an end within the next small time increment of length $d$ given that the lifetime has exceeded $x$ so far, namely

$$
\begin{aligned}
P(x<X \leq x+d \mid X>x) & =\frac{P(x<X \leq x+d)}{P(X>x)} \\
& =\frac{F(x+d)-F(x)}{1-F(x)} \\
& \approx \frac{d \times f(x)}{1-F(x)}=d \times h(x) .
\end{aligned}
$$

In the case of the Weibull distribution we have

$$
h(x)=\frac{f_{\alpha, \beta}(x)}{1-F_{\alpha, \beta}(x)}=\frac{\beta}{\alpha}\left(\frac{x}{\alpha}\right)^{\beta-1} .
$$

Various other terms are used equivalently for the hazard function, such as hazard rate, failure rate (function), or force of mortality. In the case of the Weibull hazard rate function we observe that it is increasing in $x$ when $\beta>1$, decreasing in $x$ when $\beta<1$ and constant when $\beta=1$ (exponential distribution with memoryless property).

When $\beta>1$ the part or system, for which the lifetime is modeled by a Weibull distribution, is subject to aging in the sense that an older system has a higher chance of failing during the next small time increment $d$ than a younger system.
For $\beta<1$ (less common) the system has a better chance of surviving the next small time increment $d$ as it gets older, possibly due to hardening, maturing, or curing. Often one refers to this situation as one of infant mortality, i.e., after initial early failures the survival gets better with age. However, one should keep in mind that we may be modeling parts or systems that consist of a mixture of defective or weak parts and of parts that practically can live forever. A Weibull distribution with $\beta<1$ may not do full justice to such a mixture distribution. When Weibull analysis indicates $\beta<1$ one should pay especially close attention to data quality. Often enough the following situation has been encountered. An aircraft part shows unexpected early failures and was subsequently improved/fixed by replacing it with a new part under a different part number. Lumping these early failures together with subsequent ones just because they related to the "same" functional part can lead to finding $\beta<1$.

For $\beta=1$ there is no aging, i.e., the system is as good as new given that it has survived beyond $x$, since for $\beta=1$ we have

$$
\begin{aligned}
P(X>x+h \mid X>x) & =\frac{P(X>x+h)}{P(X>x)} \\
& =\frac{\exp (-(x+h) / \alpha)}{\exp (-x / \alpha)} \\
& =\exp (-h / \alpha)=P(X>h),
\end{aligned}
$$

i.e., it is again exponential with same mean $\alpha$. One also refers to this as a random failure model in the sense that failures are due to external shocks that follow a Poisson process with rate $\lambda=1 / \alpha$. The random times between shocks are exponentially distributed with mean $\alpha$. Given that there are $k$ such shock events in an interval $[0, T]$ one can view the $k$ occurrence times as being uniformly distributed over the interval $[0, T]$, hence the allusion to random failures.

\section{Location-Scale Property of $\log (X)$}

Another useful property, of which we will make strong use, is the following location-scale property of the logtransformed Weibull distribution. By that we mean that: $X \sim \mathscr{W}(\alpha, \beta) \Longrightarrow \log (X)=Y$ has a location-scale distribution, namely its cumulative distribution function (cdf) is

$$
\begin{aligned}
P(Y & \leq y)=P(\log (X) \leq y)=P(X \leq \exp (y)) \\
& =1-\exp \left[-\left(\frac{\exp (y)}{\alpha}\right)^{\beta}\right] \\
& =1-\exp [-\exp \{(y-\log (\alpha)) \times \beta\}] \\
& =1-\exp \left[-\exp \left(\frac{y-\log (\alpha)}{1 / \beta}\right)\right]=1-\exp \left[-\exp \left(\frac{y-u}{b}\right)\right]
\end{aligned}
$$

with location parameter $u=\log (\alpha)$ and scale parameter $b=$ $1 / \beta$. The reason for referring to such parameters this way is 
Figure 2 - Ernst Hjalmar Waloddi Weibull

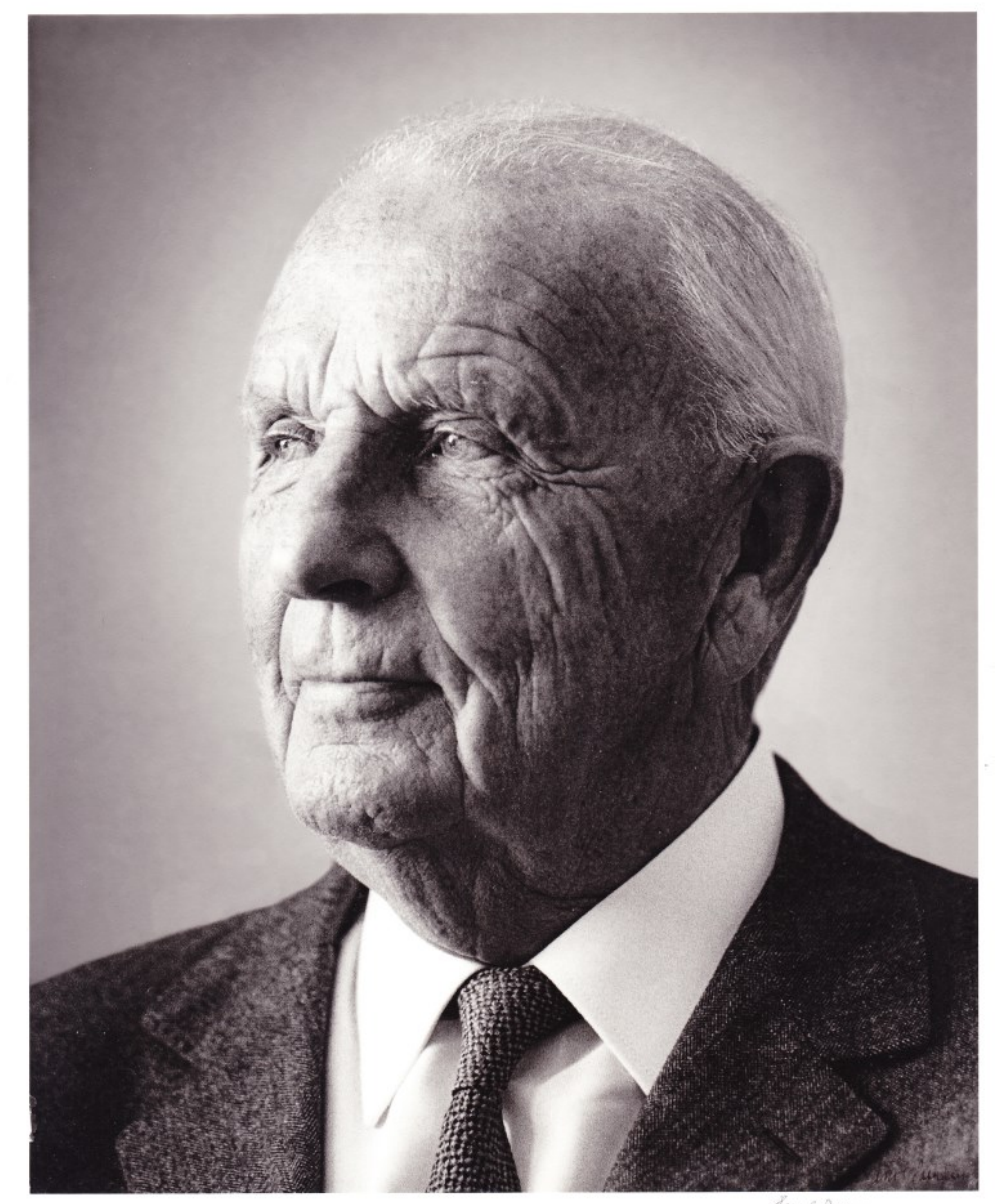

the following. If $Z \sim G(z)$ then $Y=\mu+\sigma Z \sim G((y-\mu) / \sigma)$ since

$$
\begin{aligned}
H(y) & =P(Y \leq y)=P(\mu+\sigma Z \leq y) \\
& =P(Z \leq(y-\mu) / \sigma)=G((y-\mu) / \sigma)
\end{aligned}
$$

The form $Y=\mu+\sigma X$ should make clear the notion of location-scale parameter, since $Z$ has been scaled by the factor $\sigma$ and is then shifted by $\mu$. Two prominent locationscale families are

1. $Y=\mu+\sigma Z \sim \mathscr{N}\left(\mu, \sigma^{2}\right)$, where $Z \sim \mathscr{N}(0,1)$ is standard normal with cdf $G(z)=\Phi(z)$ and thus $Y$ has cdf $H(y)=\Phi((y-\mu) / \sigma)$,

2. $Y=u+b Z$ where $Z$ has the standard extreme value distribution with $\operatorname{cdf} G(z)=1-\exp (-\exp (z))$ for $z \in R$, as in our log-transformed Weibull example above.

This distribution $G$, also known as the Gumbel distribution, plays a central role in extreme value theory, see Gum- bel, 1958, Coles, 2001,Embrechts et al., 1997, Castillo, 1988. In any such a location-scale model there is a simple relationship between the $p$-quantiles of $Y$ and $Z$, namely $y_{p}=\mu+\sigma z_{p}$ in the normal model and $y_{p}=u+b w_{p}$ in the extreme value model (using the location and scale parameters $u$ and $b$ resulting from log-transformed Weibull data). We just illustrate this in the extreme value location-scale model.

$$
\begin{aligned}
p=P\left(Z \leq w_{p}\right) & =P\left(u+b Z \leq u+b w_{p}\right) \\
& =P\left(Y \leq u+b w_{p}\right) \\
& \Longrightarrow y_{p}=u+b w_{p}
\end{aligned}
$$

with $w_{p}=\log (-\log (1-p))$. Thus $y_{p}$ is a linear function of $w_{p}=\log (-\log (1-p))$, the $p$-quantile of $G$. While $w_{p}$ is known and easily computable from $p$, the same cannot be said about $y_{p}$, since it involves the typically unknown parameters $u$ and $b$. However, for appropriate $p_{i}=(i-.5) / n$ 
one can view the $i^{\text {th }}$ ordered sample value $Y_{(i)}\left(Y_{(1)} \leq \ldots \leq\right.$ $\left.Y_{(n)}\right)$ as a good approximation for $y_{p_{i}}$. Thus the plot of $Y_{(i)}$ against $w_{p_{i}}$ should look approximately linear. This is the basis for Weibull probability plotting (and the case of plotting $Y_{(i)}$ against $z_{p_{i}}$ for normal probability plotting), a very appealing graphical procedure which gives a visual impression of how well the data fit the assumed model (normal or Weibull) and which also allows for a crude estimation of the unknown location and scale parameters, since they relate to the slope and intercept of the line that may be fitted to the perceived linear point pattern.

\section{Maximum Likelihood Estimation}

There are many ways to estimate the parameters $\theta=(\alpha, \beta)$ based on a random sample $X_{1}, \ldots, X_{n} \sim \mathscr{W}(\alpha, \beta)$. Maximum likelihood estimation (MLE) is generally the most versatile and popular method. Although MLE in the Weibull case requires numerical methods and a computer, that is no longer an issue in today's computing environment. Previously, estimates that could be computed by hand had been investigated, but they are usually less efficient than mle's (estimates derived by MLE). By efficient estimates we loosely refer to estimates that have the smallest sampling variance. MLE tends to be efficient, at least in large samples. Furthermore, under regularity conditions MLE produces estimates that have an approximate normal distribution in large samples. These properties hold in particular for random samples from a 2-parameter Weibull distribution.

When $X_{1}, \ldots, X_{n} \sim F_{\theta}(x)$ with density $f_{\theta}(x)$ then the maximum likelihood estimate of $\theta$ is that value $\theta=\hat{\theta}=$ $\hat{\theta}\left(x_{1}, \ldots, x_{n}\right)$ which maximizes the likelihood

$$
L\left(x_{1}, \ldots, x_{n}, \theta\right)=\prod_{i=1}^{n} f_{\theta}\left(x_{i}\right)
$$

$\operatorname{over} \theta$, i.e., which gives highest local probability to the observed sample $\left(X_{1}, \ldots, X_{n}\right)=\left(x_{1}, \ldots, x_{n}\right)$

$$
L\left(x_{1}, \ldots, x_{n}, \hat{\theta}\right)=\sup _{\theta}\left\{\prod_{i=1}^{n} f_{\theta}\left(x_{i}\right)\right\} .
$$

Often such maximizing values $\hat{\theta}$ are unique and one can obtain them by solving, i.e.,

$$
\frac{\partial}{\partial \theta_{j}} \prod_{i=1}^{n} f_{\theta}\left(x_{i}\right)=0 \quad j=1, \ldots, k,
$$

where $k$ is the number of parameters involved in $\theta=$ $\left(\theta_{1}, \ldots, \theta_{k}\right)$. These above equations reflect the fact that a smooth function has a horizontal tangent plane at its maximum (minimum or saddle point). Thus solving such equations is necessary but not sufficient, since it still needs to be shown that it is the location of a maximum.
Since taking derivatives of a product is tedious (product rule) one usually resorts to maximizing the log of the likelihood, i.e.,

$$
\ell\left(x_{1}, \ldots, x_{n}, \theta\right)=\log \left(L\left(x_{1}, \ldots, x_{n}, \theta\right)\right)=\sum_{i=1}^{n} \log \left(f_{\theta}\left(x_{i}\right)\right)
$$

since the value of $\theta$ that maximizes $L\left(x_{1}, \ldots, x_{n}, \theta\right)$ is the same as the value that maximizes $\ell\left(x_{1}, \ldots, x_{n}, \theta\right)$, i.e.,

$$
\ell\left(x_{1}, \ldots, x_{n}, \hat{\theta}\right)=\sup _{\theta}\left\{\sum_{i=1}^{n} \log \left(f_{\theta}\left(x_{i}\right)\right)\right\} .
$$

It is a lot simpler to deal with the likelihood equations

$$
\begin{aligned}
\frac{\partial}{\partial \theta_{j}} \ell\left(x_{1}, \ldots, x_{n}, \hat{\theta}\right) & =\frac{\partial}{\partial \theta_{j}} \sum_{i=1}^{n} \log \left(f_{\theta}\left(x_{i}\right)\right) \\
& =\sum_{i=1}^{n} \frac{\partial}{\partial \theta_{j}} \log \left(f_{\theta}\left(x_{i}\right)\right)=0 \quad j=1, \ldots, k
\end{aligned}
$$

when solving for $\theta=\hat{\theta}=\hat{\theta}\left(x_{1}, \ldots, x_{n}\right)$.

In the case of a normal random sample we have $\theta=$ $(\mu, \sigma)$ with $k=2$ and the unique solution of the likelihood equations results in the explicit expressions

$$
\hat{\mu}=\bar{x}=\sum_{i=1}^{n} x_{i} / n \quad \text { and } \quad \hat{\sigma}=\sqrt{\sum_{i=1}^{n}\left(x_{i}-\bar{x}\right)^{2} / n}
$$

and thus $\hat{\theta}=(\hat{\mu}, \hat{\sigma})$.

In the case of a Weibull sample we take the further simplifying step of dealing with the log-transformed sample $\left(y_{1}, \ldots, y_{n}\right)=\left(\log \left(x_{1}\right), \ldots, \log \left(x_{n}\right)\right)$. Recall that $Y_{i}=\log \left(X_{i}\right)$ has $\operatorname{cdf} F(y)=1-\exp (-\exp ((x-u) / b))=G((y-u) / b)$ with $G(z)=1-\exp (-\exp (z))$ with $g(z)=G^{\prime}(z)=\exp (z-\exp (z))$. Thus

$$
\left.f(y)=F^{\prime}(y)=\frac{d}{d y} F(y)=\frac{1}{b} g((y-u) / b)\right)
$$

with

$$
\log (f(y))=-\log (b)+\frac{y-u}{b}-\exp \left(\frac{y-u}{b}\right) .
$$

As partial derivatives of $\log (f(y))$ with respect to $u$ and $b$ we get

$$
\begin{aligned}
& \frac{\partial}{\partial u} \log (f(y))=-\frac{1}{b}+\frac{1}{b} \exp \left(\frac{y-u}{b}\right) \\
& \frac{\partial}{\partial b} \log (f(y))=-\frac{1}{b}-\frac{1}{b} \frac{y-u}{b}+\frac{1}{b} \frac{y-u}{b} \exp \left(\frac{y-u}{b}\right)
\end{aligned}
$$

and thus as likelihood equations 


$$
\begin{aligned}
& 0=-\frac{n}{b}+\frac{1}{b} \sum_{i=1}^{n} \exp \left(\frac{y_{i}-u}{b}\right) \quad \text { or } \quad \sum_{i=1}^{n} \exp \left(\frac{y_{i}-u}{b}\right)=n \quad \text { or } \quad \exp (u)=\left[\frac{1}{n} \sum_{i=1}^{n} \exp \left(\frac{y_{i}}{b}\right)\right]^{b}, \\
& 0=-\frac{n}{b}-\frac{1}{b} \sum_{i=1}^{n} \frac{y_{i}-u}{b}+\frac{1}{b} \sum_{i=1}^{n} \frac{y_{i}-u}{b} \exp \left(\frac{y_{i}-u}{b}\right) .
\end{aligned}
$$

i.e., we have a solution $u=\hat{u}$ once we have a solution $b=\hat{b}$. Substituting this expression for $\exp (u)$ into the second likelihood equation we get (after some cancelation and manipulation)

$$
0=\frac{\sum_{i=1}^{n} y_{i} \exp \left(y_{i} / b\right)}{\sum_{i=1}^{n} \exp \left(y_{i} / b\right)}-b-\frac{1}{n} \sum_{i=1}^{n} y_{i}
$$

Analyzing the solvability of this equation is more convenient in terms of $\beta=1 / b$ and we thus write

$$
0=\sum_{i=1}^{n} y_{i} w_{i}(\beta)-\frac{1}{\beta}-\bar{y}
$$

where

$$
w_{i}(\beta)=\frac{\exp \left(y_{i} \beta\right)}{\sum_{j=1}^{n} \exp \left(y_{j} \beta\right)}
$$

with $\sum_{i=1}^{n} w_{i}(\beta)=1$. Note that the derivative of these weights with respect to $\beta$ take the following form

$$
w_{i}^{\prime}(\beta)=\frac{d}{d \beta} w_{i}(\beta)=y_{i} w_{i}(\beta)-w_{i}(\beta) \sum_{j=1}^{n} y_{j} w_{j}(\beta) .
$$

Hence

$$
\begin{gathered}
\frac{d}{d \beta}\left\{\sum_{i=1}^{n} y_{i} w_{i}(\beta)-\frac{1}{\beta}-\bar{y}\right\}=\sum_{i=1}^{n} y_{i} w_{i}^{\prime}(\beta)+\frac{1}{\beta^{2}} \\
=\sum_{i=1}^{n} y_{i}^{2} w_{i}(\beta)-\left(\sum_{j=1}^{n} y_{j} w_{j}(\beta)\right)^{2}+\frac{1}{\beta^{2}}>0
\end{gathered}
$$

since

$$
\begin{aligned}
\operatorname{var}_{w}(y) & =\sum_{i=1}^{n} y_{i}^{2} w_{i}(\beta)-\left(\sum_{j=1}^{n} y_{j} w_{j}(\beta)\right)^{2} \\
& =E_{w}\left(y^{2}\right)-\left[E_{w}(y)\right]^{2} \geq 0
\end{aligned}
$$

can be interpreted as a variance of the $n$ values of $y=$ $\left(y_{1}, \ldots, y_{n}\right)$ with weights or probabilities given by $w=$ $\left(w_{1}(\beta), \ldots, w_{n}(\beta)\right)$. Thus the reduced second likelihood equation $\sum y_{i} w_{i}(\beta)-1 / \beta-\bar{y}=0$ has a unique solution (if it has a solution at all) since the equation's left side is strictly increasing.
Note that $w_{i}(\beta) \rightarrow 1 / n$ as $\beta \rightarrow 0$. Thus $\sum y_{i} w_{i}(\beta)-1 / \beta-$ $\bar{y} \approx-1 / \beta \rightarrow-\infty$ as $\beta \rightarrow 0$.

Furthermore, with $M=\max \left(y_{1}, \ldots, y_{n}\right)$ and $\beta \rightarrow \infty$ we have

$$
w_{i}(\beta)=\exp \left(\beta\left(y_{i}-M\right)\right) / \sum_{j=1}^{n} \exp \left(\beta\left(y_{j}-M\right)\right)
$$

when $y_{i}<M$ and $w_{i}(\beta) \rightarrow 1 / r$ when $y_{i}=M$ where $r \geq 1$ is the number of $y_{i}$ coinciding with $M$. Thus

$$
\sum y_{i} w_{i}(\beta)-1 / \beta-\bar{y} \approx M-1 / \beta-\bar{y} \rightarrow M-\bar{y}>0
$$

as $\beta \rightarrow \infty$ where $M-\bar{y}>0$ assumes that not all $y_{i}$ coincide (a degenerate case with probability 0 ). That this unique solution corresponds to a maximum and thus a unique global maximum takes some extra effort and we refer to Scholz, 1996 (revised 2001) for an even more general treatment that covers Weibull analysis with right censored data and covariates.

However, a somewhat loose argument can be given as follows. If we consider the likelihood of the logtransformed Weibull data we have

$$
L\left(y_{1}, \ldots, y_{n}, u, b\right)=\frac{1}{b^{n}} \prod_{i=1}^{n} g\left(\frac{y_{i}-u}{b}\right) .
$$

Contemplate this likelihood for fixed $y=\left(y_{1}, \ldots, y_{n}\right)$ and for parameters $u$ with $|u| \rightarrow \infty$ (the location moves away from all observed data values $y_{1}, \ldots, y_{n}$ ) and $b$ with $b \rightarrow 0$ (the spread becomes very concentrated on some point and cannot simultaneously do so at all values $y_{1}, \ldots, y_{n}$, unless they are all the same, excluded as a zero probability degeneracy) and $b \rightarrow \infty$ (in which case all probability is diffused thinly over the whole half plane $\{(u, b): u \in R, b>0\})$, it is then easily seen that this likelihood approaches zero in all cases. Since this likelihood is positive everywhere (but approaching zero near the fringes of the parameter space, the above half plane) it follows that it must have a maximum somewhere with zero partial derivatives. We showed there is only one such point (uniqueness of the solution to the likelihood equations) and thus there can only be one unique (global) maximum, which then is also the unique maximum likelihood estimate $\hat{\theta}=(\hat{u}, \hat{b})$. 
In solving $0=\sum y_{i} \exp \left(y_{i} / b\right) / \sum \exp \left(y_{i} / b\right)-b-\bar{y}$, it is numerically advantageous to solve the equivalent equation $0=\sum y_{i} \exp \left(\left(y_{i}-M\right) / b\right) / \sum \exp \left(\left(y_{i}-M\right) / b\right)-b-\bar{y}$ where $M=\max \left(y_{1}, \ldots, y_{n}\right)$. This avoids overflow or accuracy loss in the exponentials when the $y_{i}$ tend to be large.

The above derivations go through with very little change when instead of observing a full sample $Y_{1}, \ldots, Y_{n}$ we only observe the $r \geq 2$ smallest sample values $Y_{(1)}<$ $\ldots<Y_{(r)}$. Such data is referred to as type II censored data. This situation typically arises in a laboratory setting when several units are put on test (subjected to failure exposure) simultaneously and the test is terminated (or evaluated) when the first $r$ units have failed. In that case we know the first $r$ failure times $X_{(1)}<\ldots<X_{(r)}$ and thus $Y_{(i)}=$ $\log \left(X_{(i)}\right), i=1, \ldots, r$, and we know that the lifetimes of the remaining units exceed $X_{(r)}$ or that $Y_{(i)}>Y_{(r)}$ for $i>r$. The advantage of such data collection is that we do not have to wait until all $n$ units have failed. Furthermore, if we put a lot of units on test (high $n$ ) we increase our chance of seeing our first $r$ failures before a fixed time $y$. This is a simple consequence of the following binomial probability statement:

$$
\begin{aligned}
P\left(Y_{(r)} \leq y\right) & =P(\text { at least } r \text { failures } \leq y \text { in } n \text { trials }) \\
& =\sum_{i=r}^{n}\left(\begin{array}{c}
n \\
i
\end{array}\right) P(Y \leq y)^{i}(1-P(Y \leq y))^{n-i}
\end{aligned}
$$

which is strictly increasing in $n$ for any fixed $y$ and $r \geq 1$.

The joint density of $Y_{(1)}, \ldots, Y_{(n)}$ at $\left(y_{1}, \ldots, y_{n}\right)$ with $y_{1}<$ $\ldots<y_{n}$ is

$$
f\left(y_{1}, \ldots, y_{n}\right)=n ! \prod_{i=1}^{n} \frac{1}{b} g\left(\frac{y_{i}-u}{b}\right)=n ! \prod_{i=1}^{n} f\left(y_{i}\right)
$$

where the multiplier $n$ ! just accounts for the fact that all $n$ ! permutations of $y_{1}, \ldots, y_{n}$ could have been the order in which these values were observed and all of these orders have the same density (probability). Integrating out $y_{n}>y_{n-1}>\ldots>y_{r+1}\left(>y_{r}\right)$ and using $\bar{F}(y)=1-F(y)$ we get after $n-r$ successive integration steps the joint density of the first $r$ failure times $y_{1}<\ldots<y_{r}$ as

$$
\begin{aligned}
& f\left(y_{1}, \ldots, y_{n-1}\right)=n ! \prod_{i=1}^{n-1} f\left(y_{i}\right) \times \int_{y_{n-1}}^{\infty} f\left(y_{n}\right) d y_{n} \\
& =n ! \prod_{i=1}^{n-1} f\left(y_{i}\right) \bar{F}\left(y_{n-1}\right) \\
& f\left(y_{1}, \ldots, y_{n-2}\right)=n ! \prod_{i=1}^{n-2} f\left(y_{i}\right) \times \int_{y_{n-2}}^{\infty} f\left(y_{n-1}\right) \bar{F}\left(y_{n-1}\right) d y_{n-1} \\
& =n ! \prod_{i=1}^{n-2} f\left(y_{i}\right) \times \frac{1}{2} \bar{F}^{2}\left(y_{n-2}\right) \\
& f\left(y_{1}, \ldots, y_{n-3}\right)=n ! \prod_{i=1}^{n-3} f\left(y_{i}\right) \times \int_{y_{n-3}}^{\infty} f\left(y_{n-2}\right) \bar{F}^{2}\left(y_{n-2}\right) / 2 d y_{n-2} \\
& =n ! \prod_{i=1}^{n-3} f\left(y_{i}\right) \times \frac{1}{3 !} \bar{F}^{3}\left(y_{n-3}\right) \\
& \text {... } \\
& f\left(y_{1}, \ldots, y_{r}\right)=n ! \prod_{i=1}^{r} f\left(y_{i}\right) \times \frac{1}{(n-r) !} \bar{F}^{n-r}\left(y_{r}\right) \\
& =\left[\frac{n !}{(n-r) !} \prod_{i=1}^{r} f\left(y_{i}\right)\right] \times\left[1-F\left(y_{r}\right)\right]^{n-r} \\
& =r ! \prod_{i=1}^{r} \frac{1}{b} g\left(\frac{y_{i}-u}{b}\right) \times\left(\begin{array}{l}
n \\
r
\end{array}\right)\left[1-G\left(\frac{y_{r}-u}{b}\right)\right]^{n-r}
\end{aligned}
$$

with log-likelihood

$\ell\left(y_{1}, \ldots, y_{r}, u, b\right)=\log \left(\frac{n !}{(n-r) !}\right)-r \log (b)+\sum_{i=1}^{r} \frac{y_{i}-u}{b}-\sum_{i=1}^{r} \star \exp \left(\frac{y_{i}-u}{b}\right)$

where we use the notation

$$
\sum_{i=1}^{r} \star x_{i}=\sum_{i=1}^{r} x_{i}+(n-r) x_{r}
$$

The likelihood equations are

$$
\begin{aligned}
& 0=\frac{\partial}{\partial u} \ell\left(y_{1}, \ldots, y_{r}, u, b\right)=-\frac{r}{b}+\frac{1}{b} \sum_{i=1}^{r} \star \exp \left(\frac{y_{i}-u}{b}\right) \quad \text { or } \quad \exp (u)=\left[\frac{1}{r} \sum_{i=1}^{r} \star \exp \left(\frac{y_{i}}{b}\right)\right]^{b} \\
& 0=\frac{\partial}{\partial b} \ell\left(y_{1}, \ldots, y_{r}, u, b\right)=-\frac{r}{b}-\frac{1}{b} \sum_{i=1}^{r} \frac{y_{i}-u}{b}+\frac{1}{b} \sum_{i=1}^{r} \star \frac{y_{i}-u}{b} \exp \left(\frac{y_{i}-u}{b}\right)
\end{aligned}
$$


where again the transformed first equation gives us a solution $\hat{u}$ once we have a solution $\hat{b}$ for $b$. Using this in the second equation, it transforms to a single equation in $b$ alone, namely

$$
\frac{\sum_{i=1}^{r}{ }^{\star} y_{i} \exp \left(y_{i} / b\right)}{\sum_{i=1}^{r}{ }^{\star} \exp \left(y_{i} / b\right)}-b-\frac{1}{r} \sum_{i=1}^{r} y_{i}=0 .
$$

Again it is advisable to use the equivalent but computationally more stable form

$$
\frac{\sum_{i=1}^{r}{ }^{\star} y_{i} \exp \left(\left(y_{i}-y_{r}\right) / b\right)}{\sum_{i=1}^{r}{ }^{\star} \exp \left(\left(y_{i}-y_{r}\right) / b\right)}-b-\frac{1}{r} \sum_{i=1}^{r} y_{i}=0 .
$$

As in the complete sample case one sees that this equation has a unique solution $\hat{b}$ and that $(\hat{u}, \hat{b})$ gives the location of the (unique) global maximimum of the likelihood function, i.e., $(\hat{u}, \hat{b})$ are the mle's.

\section{Computation of Maximum Likelihood Estimates in $\mathrm{R}$}

The computation of the mle's of the Weibull parameters $\alpha$ and $\beta$ is facilitated by the function survreg which is part of the R package survival. Here survreg is used in its most basic form in the context of Weibull data (full sample or type II censored Weibull data). survreg does a whole lot more than compute the mle's but we will not deal with these aspects here, at least for now. Listing 1 gives an $\mathrm{R}$ function, called Weibull.mle, that uses survreg to compute these estimates. Note that it tests for the existence of survreg before calling it. This function is part of the Weibull R functions that accompany this article.

Note that survreg analyzes objects of class Surv. Here such an object is created by the function Surv and it basically adjoins the failure times with a status vector of same length. The status is 1 when a time corresponds to an actual failure time. It is 0 when the corresponding time is a censoring time, i.e., we only know that the unobserved actual failure time exceeds the reported censoring time. In the case of type II censored data these censoring times equal the $r^{\text {th }}$ largest failure time.

To get a sense of the calculation speed of this function we ran Weibull.mle a 1000 times, which tells us that the time to compute the mle's in a sample of size $n=10$ is roughly $1.53 / 1000=.00153$. This fact plays a significant role later on in the various inference procedures which we will discuss.

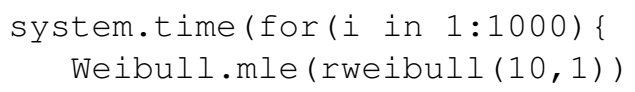

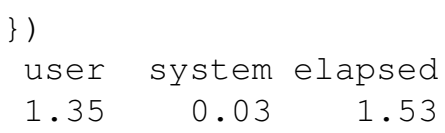

These results were obtained with an Intel(R) Core(TM) i5-4460 $3.20 \mathrm{GHz}$ CPU with 16 GB RAM. For $n=100,500,1000,5000$ the elapsed times came to $1.71,3.47,7.15$ and 26.68, respectively. The relationship of computing time to $n$ appears to be quite linear, as Figure 3 shows, produced by timing.plot ( ).

\section{Location and Scale Equivariance of Maximum Likelihood Estimates}

The maximum likelihood estimates $\hat{u}$ and $\hat{b}$ of the location and scale parameters $u$ and $b$ have the following equivariance properties which will play a strong role in the later pivot construction and resulting confidence intervals.

Based on data $\mathbf{z}=\left(z_{1}, \ldots, z_{n}\right)$ we denote the estimates of $u$ and $b$ more explicitly by $\hat{u}\left(z_{1}, \ldots, z_{n}\right)=\hat{u}(\mathbf{z})$ and $\hat{b}\left(z_{1}, \ldots, z_{n}\right)=\hat{b}(\mathbf{z})$. If we transform $\mathbf{z}$ to $\mathbf{r}=\left(r_{1}, \ldots, r_{n}\right)$ with $r_{i}=A+B z_{i}$, where $A \in R$ and $B>0$ are arbitrary constant, then

$$
\hat{u}\left(r_{1}, \ldots, r_{n}\right)=A+B \hat{u}\left(z_{1}, \ldots, z_{n}\right)
$$

or

$$
\hat{u}(\mathbf{r})=\hat{u}(A+B \mathbf{z})=A+B \hat{u}(\mathbf{z})
$$

and

$$
\hat{b}\left(r_{1}, \ldots, r_{n}\right)=B \hat{b}\left(z_{1}, \ldots, z_{n}\right)
$$

or

$$
\hat{b}(\mathbf{r})=\hat{b}(A+B \mathbf{z})=B \hat{b}(\mathbf{z}) .
$$

These properties are naturally desirable for any location and scale estimates and for mle's they are indeed true.

Proof: Observe the following defining properties of the mle's in terms of $\mathbf{z}=\left(z_{1}, \ldots, z_{n}\right)$ and $\mathbf{r}=\left(r_{1}, \ldots, r_{n}\right)$

$$
\begin{gathered}
\sup _{u, b}\left\{\frac{1}{b^{n}} \prod_{i=1}^{n} g\left(\left(z_{i}-u\right) / b\right)\right\}=\frac{1}{\hat{b}^{n}(\mathbf{z})} \prod_{i=1}^{n} g\left(\left(z_{i}-\hat{u}(\mathbf{z})\right) / \hat{b}(\mathbf{z})\right) \\
\sup _{u, b}\left\{\frac{1}{b^{n}} \prod_{i=1}^{n} g\left(\left(r_{i}-u\right) / b\right)\right\} \\
=\frac{1}{\hat{b}^{n}(\mathbf{r})} \prod_{i=1}^{n} g\left(\left(r_{i}-\hat{u}(\mathbf{r})\right) / \hat{b}(\mathbf{r})\right) \\
=\frac{1}{B^{n}} \frac{1}{(\hat{b}(\mathbf{r}) / B)^{n}} \prod_{i=1}^{n} g\left(\left(z_{i}-(\hat{u}(\mathbf{r})-A) / B\right) /(\hat{b}(\mathbf{r}) / B)\right)
\end{gathered}
$$

but also 
Figure 3 - Weibull Parameter MLE Computation Time in Relation to Sample Size $n$

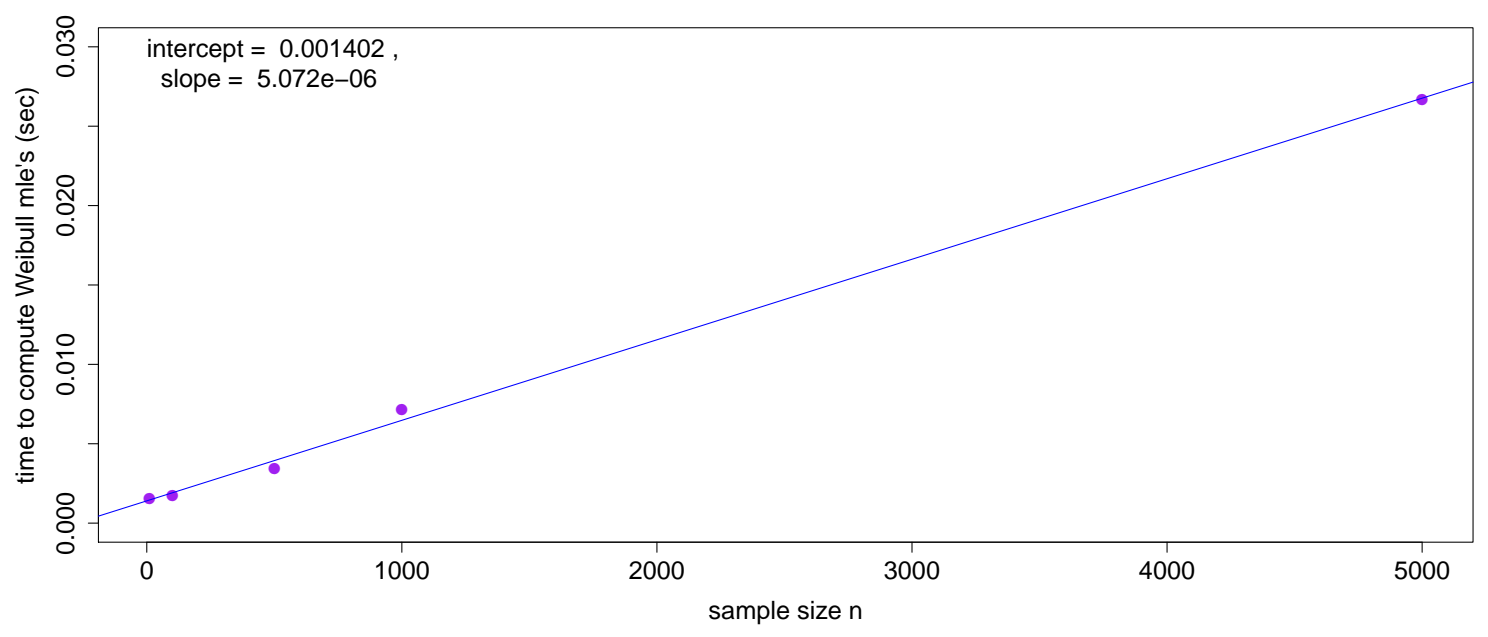

$$
\begin{aligned}
\sup _{u, b}\left\{\frac{1}{b^{n}} \prod_{i=1}^{n} g\left(\left(r_{i}-u\right) / b\right)\right\} & =\sup _{u, b}\left\{\frac{1}{b^{n}} \prod_{i=1}^{n} g\left(\left(A+B z_{i}-u\right) / b\right)\right\} \\
& =\sup _{u, b}\left\{\frac{1}{B^{n}} \frac{1}{(b / B)^{n}} \prod_{i=1}^{n} g\left(\left(z_{i}-(u-A) / B\right) /(b / B)\right)\right\} \\
\tilde{u}=(u-A) / B \Rightarrow & =\sup _{\tilde{b}=b / B}\left\{\frac{1}{B^{n}} \frac{1}{\tilde{b}^{n}} \prod_{i=1}^{n} g\left(\left(z_{i}-\tilde{u}\right) / \tilde{b}\right)\right\}=\frac{1}{B^{n}} \frac{1}{\hat{b}^{n}(\mathbf{z})} \prod_{i=1}^{n} g\left(\left(z_{i}-\hat{u}(\mathbf{z})\right) / \hat{b}(\mathbf{z})\right)
\end{aligned}
$$

Thus by the uniqueness of the mle's we have

$$
\hat{u}(\mathbf{z})=(\hat{u}(\mathbf{r})-A) / B \quad \text { and } \quad \hat{b}(\mathbf{z})=\hat{b}(\mathbf{r}) / B
$$

or

$$
\hat{u}(\mathbf{r})=\hat{u}(A+B \mathbf{z})=A+B \hat{u}(\mathbf{z})
$$

and

$$
\hat{b}(\mathbf{r})=\hat{b}(A+B \mathbf{z})=B \hat{b}(\mathbf{z}) \quad \text { q.e.d. }
$$

The same equivariance properties hold for the mle's in the context of type II censored samples, as is easily verified.

Tests of Fit Based on the Empirical Distribution Function

Relying on subjective assessment of linearity in Weibull probability plots in order to judge whether a sample comes from a 2-parameter Weibull population takes a fair amount of experience. It is simpler and more objective to employ a formal test of fit which compares the empirical distribution function $\hat{F}_{n}(x)$ of a sample with the fitted Weibull distribution function $\hat{F}(x)=F_{\hat{\alpha}, \hat{\beta}}(x)$ using one of several common discrepancy metrics.

The empirical distribution function (EDF) of a sample $X_{1}, \ldots, X_{n}$ is defined as

$$
\hat{F}_{n}(x)=\frac{\text { \# of observations } \leq x}{n}=\frac{1}{n} \sum_{i=1}^{n} I_{\left\{X_{i} \leq x\right\}}
$$

where $I_{A}=1$ when $A$ is true, and $I_{A}=0$ when $A$ is false. The fitted Weibull distribution function (using mle's $\hat{\alpha}$ and $\hat{\beta}$ ) is

$$
\hat{F}(x)=F_{\hat{\alpha}, \hat{\beta}}(x)=1-\exp \left(-\left(\frac{x}{\hat{\alpha}}\right)^{\hat{\beta}}\right) .
$$

From the law of large numbers (LLN) we see that for any $x$ we have that $\hat{F}_{n}(x) \longrightarrow F_{\alpha, \beta}(x)$ as $n \longrightarrow \infty$, provided the 
random sample $X_{1}, \ldots, X_{n} \sim \mathscr{W}(\alpha, \beta)$. Just view $\hat{F}_{n}(x)$ as a binomial proportion or as an average of Bernoulli random variables.

From MLE theory we also know that $\hat{F}(x)=F_{\hat{\alpha}, \hat{\beta}}(x) \longrightarrow$ $F_{\alpha, \beta}(x)$ as $n \longrightarrow \infty$ (also derived from the LLN).

Since the limiting $\operatorname{cdf} F_{\alpha, \beta}(x)$ is continuous in $x$ one can argue that these convergence statements can be made uniformly in $x$, i.e.,

$\sup _{x}\left|\hat{F}_{n}(x)-F_{\alpha, \beta}(x)\right| \longrightarrow 0$ and $\sup _{x}\left|F_{\hat{\alpha}, \hat{\beta}}(x)-F_{\alpha, \beta}(x)\right| \longrightarrow 0$

as $n \longrightarrow \infty$ and thus

$$
\sup _{x}\left|\hat{F}_{n}(x)-F_{\hat{\alpha}, \hat{\beta}}(x)\right| \longrightarrow 0
$$

as $n \longrightarrow \infty$ for all $\alpha>0$ and $\beta>0$. The distance

$$
D_{\mathrm{KS}}(F, G)=\sup _{x}|F(x)-G(x)|
$$

is known as the Kolmogorov-Smirnov distance between two cdf's $F$ and $G$.

Figures 4 and 5 give illustrations of this KolmogorovSmirnov distance between EDF and fitted Weibull distribution and show the relationship between sampled true Weibull distribution, fitted Weibull distribution, and empirical distribution function. These plots were generated by the supplied function edf.plot using edf.plot ( $\mathrm{n}$ $=10$, alpha $=10000$, beta $=2$ ) and $\mathrm{n}=20$, 50 and 100.

Some comments:

1. It can be noted that the closeness between $\hat{F}_{n}(x)$ and $F_{\hat{\alpha}, \hat{\beta}}(x)$ is usually more pronounced than their respective closeness to $F_{\alpha, \beta}(x)$, in spite of the sequence of the above convergence statements.

2. This can be understood from the fact that both $\hat{F}_{n}(x)$ and $F_{\hat{\alpha}, \hat{\beta}}(x)$ fit the data, i.e., try to give a good representation of the data. The fit of the true distribution, although being the origin of the data, is not always good due to sampling variation.

3. The closeness between all three distributions improves as $n$ gets larger.

Several other distances between cdf's $F$ and $G$ have been proposed and investigated in the literature, see Stephens, 1986. We will only discuss two of them, the Cramér-von Mises distance $D_{\mathrm{CvM}}$ and the AndersonDarling distance $D_{\mathrm{AD}}$. They are defined respectively as follows

$$
\begin{aligned}
D_{\mathrm{CvM}}(F, G) & =\int_{-\infty}^{\infty}(F(x)-G(x))^{2} d G(x) \\
& =\int_{-\infty}^{\infty}(F(x)-G(x))^{2} g(x) d x
\end{aligned}
$$

and

$$
\begin{aligned}
D_{\mathrm{AD}}(F, G) & =\int_{-\infty}^{\infty} \frac{(F(x)-G(x))^{2}}{G(x)(1-G(x))} d G(x) \\
& =\int_{-\infty}^{\infty} \frac{(F(x)-G(x))^{2}}{G(x)(1-G(x))} g(x) d x .
\end{aligned}
$$

Rather than focussing on the very local phenomenon of a maximum discrepancy at some point $x$ as in $D_{\mathrm{KS}}$, these alternate distances or discrepancy metrics integrate these distances in squared form over all $x$, weighted by $g(x)$ in the case of $D_{\mathrm{CvM}}(F, G)$ and by $g(x) /[G(x)(1-G(x))]$ in the case $D_{\mathrm{AD}}(F, G)$. In the latter case, the denominator increases the weight in the tails of the $G$ distribution, i.e., compensates to some extent for the tapering off in the density $g(x)$. Thus $D_{\mathrm{AD}}(F, G)$ is favored in situations where judging tail behavior is important, e.g., in risk situations. Because of the integration nature of these last two metrics they have more global character. There is no easy graphical representation of these metrics, except to suggest that when viewing the previous figures illustrating $D_{\mathrm{KS}}$ one should look at all vertical distances (large and small) between $\hat{F}_{n}(x)$ and $\hat{F}(x)$, square them and accumulate these squares in the appropriately weighted fashion. For example, when one cdf is shifted relative to the other by a small amount (no large vertical discrepancy), these small vertical discrepancies (squared) will add up and indicate a moderately large difference between the two compared cdf's.

We point out the asymmetric nature of these last two metrics, i.e., we typically have

$D_{\mathrm{CvM}}(F, G) \neq D_{\mathrm{CvM}}(G, F) \quad$ and $\quad D_{\mathrm{AD}}(F, G) \neq D_{\mathrm{AD}}(G, F)$.

When using these metrics for tests of fit one usually takes the cdf with a density (the estimated model distribution to be tested) as the one with respect to which the integration takes place, while the other cdf is taken to be the EDF.

As complicated as these metrics may look at first glance, their computation is quite simple. We will give the following computational expressions (without proof):

$$
\begin{aligned}
D_{\mathrm{KS}}\left(\hat{F}_{n}(x), \hat{F}(x)\right)=D \\
=\max \left[\max \left\{i / n-V_{(i)}\right\}, \max \left\{V_{(i)}-(i-1) / n\right\}\right]
\end{aligned}
$$

where $V_{(1)} \leq \ldots \leq V_{(n)}$ are the ordered values of $V_{i}=$ $\hat{F}\left(X_{i}\right), i=1, \ldots, n$.

For the other two test of fit criteria we have

$$
D_{\mathrm{CvM}}\left(\hat{F}_{n}(x), \hat{F}(x)\right)=W^{2}=\sum_{i=1}^{n}\left\{V_{(i)}-\frac{2 i-1}{2 n}\right\}^{2}+\frac{1}{12 n}
$$

and

$$
\begin{aligned}
D_{\mathrm{AD}}\left(\hat{F}_{n}(x), \hat{F}(x)\right) & =A^{2} \\
= & -n-\frac{1}{n} \sum_{i=1}^{n}(2 i-1)\left[\log \left(V_{(i)}\right)+\log \left(1-V_{(n-i+1)}\right)\right] .
\end{aligned}
$$

The Quantitative Methods for Psychology 
Figure 4 - Illustration of Kolmogorov-Smirnov Distance for $n=10$ and $n=20$

(a) $n=10$

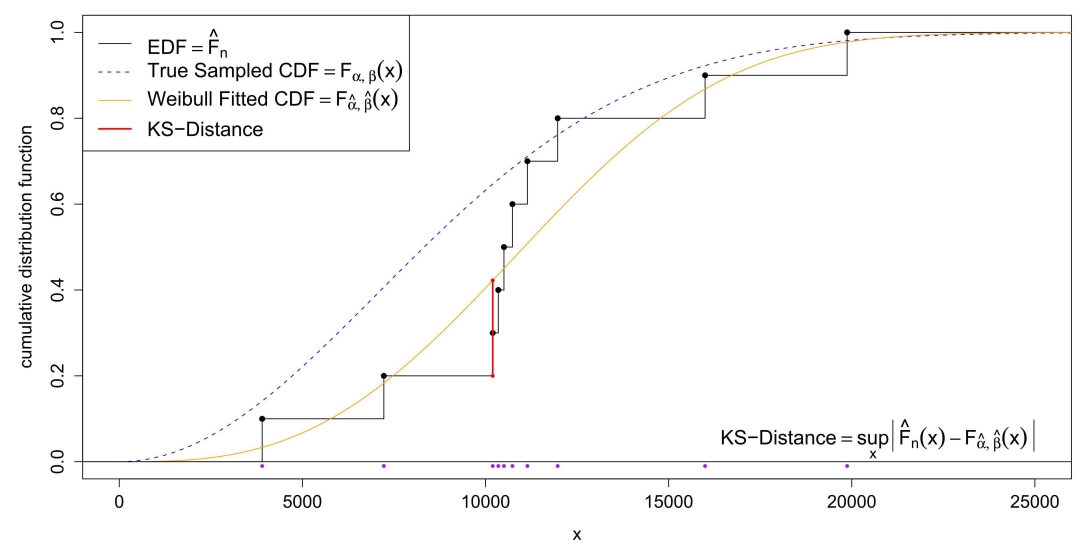

(b) $n=20$

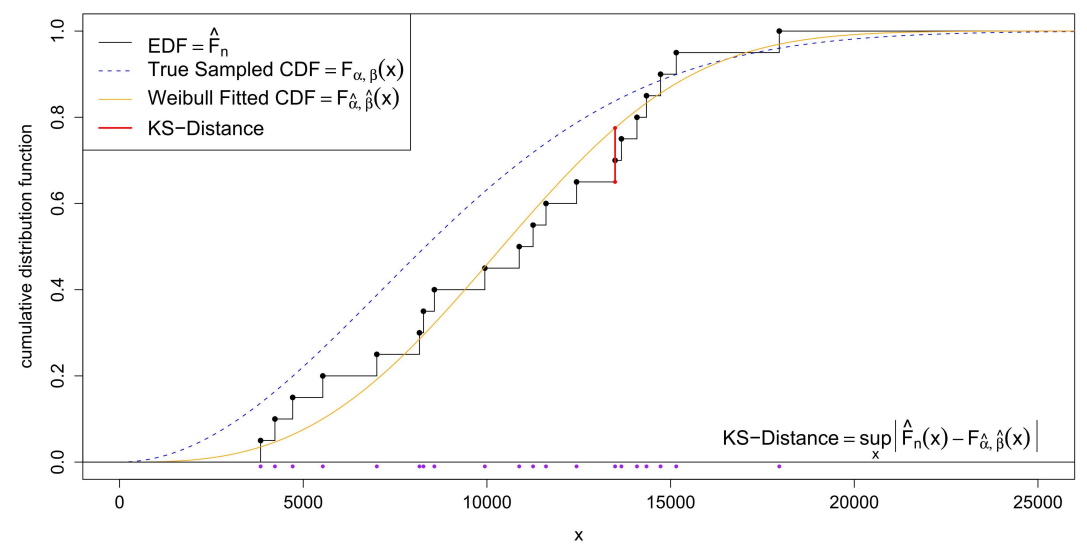

In order to carry out these tests of fit we need to know the null distributions of $D, W^{2}$ and $A^{2}$. Quite naturally we would reject the hypothesis of a sampled Weibull distribution whenever $D$ or $W^{2}$ or $A^{2}$ are too large. The null distributions of $D, W^{2}$ and $A^{2}$ do not depend on the unknown parameters $\alpha$ and $\beta$, being estimated by $\hat{\alpha}$ and $\hat{\beta}$ in $V_{i}=\hat{F}\left(X_{i}\right)=F_{\hat{\alpha}, \hat{\beta}}\left(X_{i}\right)$. The reason for this is that the $V_{i}$ have a distribution that is independent of the unknown parameters $\alpha$ and $\beta$. This is seen as follows. Using our prior notation we write $\log \left(X_{i}\right)=Y_{i}=u+b Z_{i}$ and since

$$
\begin{aligned}
F(x)=P(X \leq x) & =P(\log (X) \leq \log (x)) \\
& =P(Y \leq y)=1-\exp (-\exp ((y-u) / b))
\end{aligned}
$$

and thus

$$
\begin{aligned}
V_{i}=\hat{F}\left(X_{i}\right) & =1-\exp \left(-\exp \left(\left(Y_{i}-\hat{u}(\mathbf{Y})\right) / \hat{b}(\mathbf{Y})\right)\right) \\
& =1-\exp \left(-\exp \left(\left(u+b Z_{i}-\hat{u}(u+b \mathbf{Z})\right) / \hat{b}(u+b \mathbf{Z})\right)\right) \\
& =1-\exp \left(-\exp \left(\left(u+b Z_{i}-u-b \hat{u}(\mathbf{Z})\right) /[b \hat{b}(\mathbf{Z}])\right)\right) \\
& =1-\exp \left(-\exp \left(\left(Z_{i}-\hat{u}(\mathbf{Z})\right) / \hat{b}(\mathbf{Z})\right)\right)
\end{aligned}
$$

and all dependence on the unknown parameters $u=\log (\alpha)$ and $b=1 / \beta$ has canceled out.

This opens up the possibility of using simulations to find good approximations to these null distributions for any $n$, especially in view of the previously reported timing results for computing the mle's $\hat{\alpha}$ and $\hat{\beta}$ of $\alpha$ and $\beta$. Just generate samples $\mathbf{X}^{\star}=\left(X_{1}^{\star}, \ldots, X_{n}^{\star}\right)$ from $\mathbb{W}(\alpha=1, \beta=$ 1) (standard exponential distribution), compute the corresponding $\hat{\alpha}^{\star}=\hat{\alpha}\left(\mathbf{X}^{\star}\right)$ and $\hat{\beta}^{\star}=\hat{\beta}\left(\mathbf{X}^{\star}\right)$, then $V_{i}^{\star}=\hat{F}\left(X_{i}^{\star}\right)=$ $F_{\hat{\alpha}^{\star}, \hat{\beta}^{\star}}\left(X_{i}^{\star}\right)$ (where $F_{\alpha, \beta}(x)$ is the cdf of $\mathcal{W}(\alpha, \beta)$ ) and from that the values $D^{\star}=D\left(\mathbf{X}^{\star}\right), W^{2 \star}=W^{2}\left(\mathbf{X}^{\star}\right)$ and $A^{2 \star}=$ 
Figure 5 - Illustration of Kolmogorov-Smirnov Distance for $n=50$ and $n=100$

(a) $n=50$

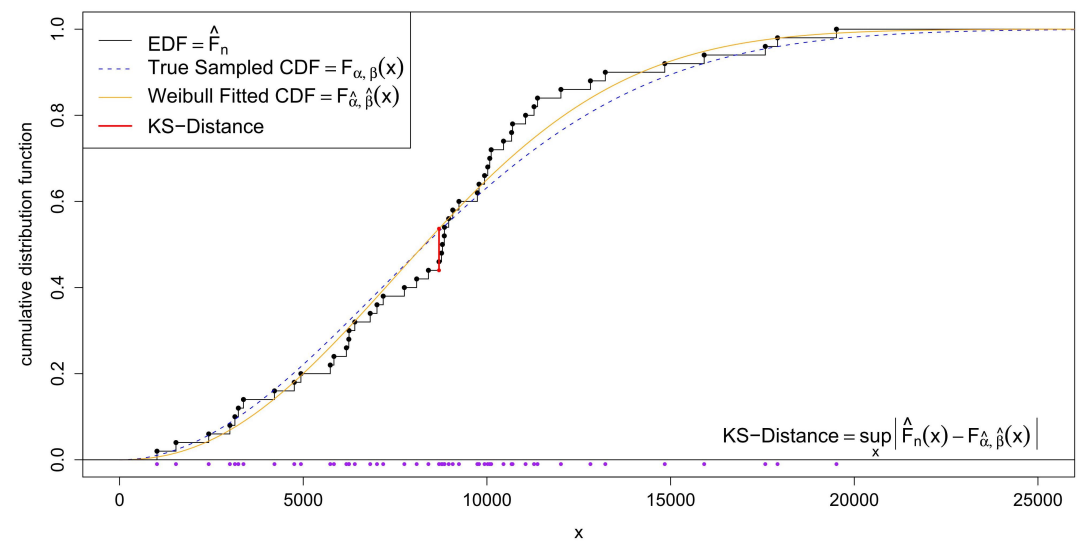

(b) $n=100$

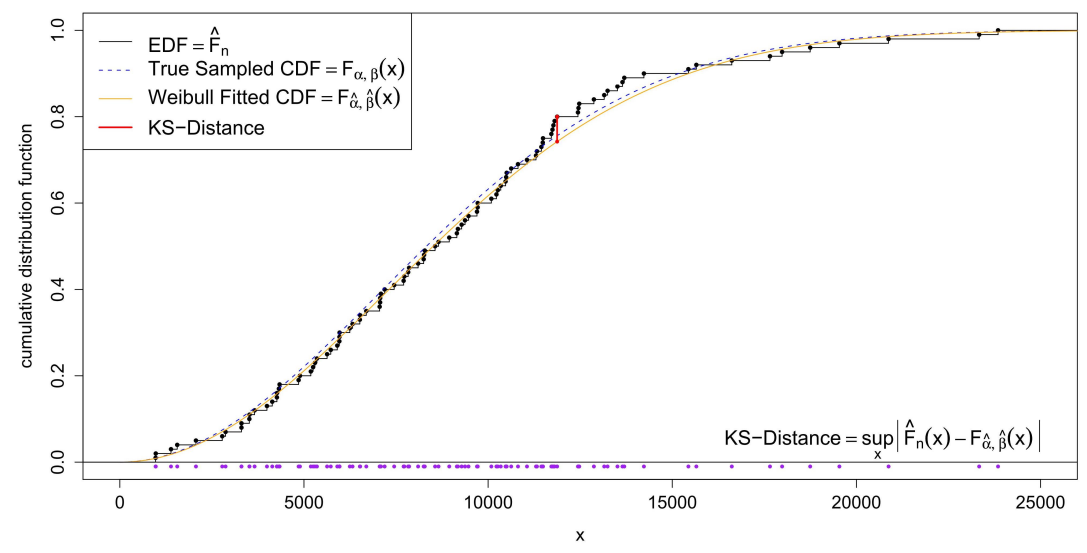

$A^{2}\left(\mathbf{X}^{\star}\right)$. Calculating all three test of fit criteria makes sense since the main calculation effort is in getting the mle's $\hat{\alpha}^{\star}$ and $\hat{\beta}^{\star}$. Repeating this a large number of times, say $N_{\text {sim }}=$ 10000 , should give us a reasonably good approximation to the desired null distribution and from it one can determine appropriate $p$-values for any sample $X_{1}, \ldots, X_{n}$ for which one wishes to assess whether the Weibull distribution hypothesis is tenable or not. If $C(\mathbf{X})$ denotes the used test of fit criterion then the estimated $p$-value of the observed sample $\mathbf{x}$ is simply the proportion of $C\left(\mathbf{X}^{\star}\right)$ that are $\geq C(\mathbf{x})$.

Prior to the ease of current computing, Stephens, 1986 provided tables for the $(1-\alpha)$-quantiles $q_{1-\alpha}$ of these null distributions. For the $n$-adjusted versions $A^{2}(1+.2 / \sqrt{n})$ and $W^{2}(1+.2 / \sqrt{n})$ these null distributions appear to be independent of $n$ and $(1-\alpha)$-quantiles were given for $\alpha=$ $.25, .10, .05, .025, .01$. Plotting $\log (\alpha /(1-\alpha))$ against $q_{1-\alpha}$ shows a mildly quadratic pattern which can be used to interpolate or extrapolate the appropriate $p$-value (observed significance level $\alpha$ ) for any observed $n$-adjusted value $A^{2}(1+.2 / \sqrt{n})$ and $W^{2}(1+.2 / \sqrt{n})$, as is illustrated in Figure 6 .

For $\sqrt{n} D$ the null distribution still depends on $n$ (in spite of the normalizing factor $\sqrt{n})$ and $(1-\alpha)$-quantiles for $\alpha=.10, .05, .025, .01$ were tabulated for $n=10,20,50, \infty$ by Stephens, 1986. Here a double inter- and extrapolation scheme is needed, first by plotting these quantiles against $1 / \sqrt{n}$, fitting quadratics in $1 / \sqrt{n}$ and reading off the four interpolated quantile values for the needed $n_{0}$ (the sample size at issue) and as a second step perform the interpolation or extrapolation scheme as it was done previously, but using a cubic this time. This is illustrated in Figure 7.

The functions for computing these $\mathrm{p}$-values (via interpolation from Stephens' tabled values) are GOF . KS . test, GOF. CVM. test, and GOF.AD. test. They compute $p$ values for $n$-adjusted test criteria $\sqrt{n} D, W^{2}(1+.2 / \sqrt{n})$, and $A^{2}(1+.2 / \sqrt{n})$, respectively. These functions have an op- 
tional argument graphic where graphic $=\mathrm{T}$ causes the interpolation graphs shown in Figures 6 and 7 to be produced, otherwise only the $p$-values are given. The function Weibull.GOF . test does a Weibull goodness of fit test on any given sample, returning $p$-values for all three test criteria.

One could easily reproduce and extend the tables given by Stephens (1986) so that extrapolations becomes less of an issue. For $n=100$ it should take about 17 seconds to simulate the null distributions based on $N_{\text {sim }}=10,000$ and the previously given timing of $1.71 \mathrm{sec}$ for $N_{\text {sim }}=1,000$. This timing estimate ignores the calculation of $D, W^{2}$, and $A^{2}$.

\section{Pivots}

For the following generic discussion of pivots assume that the data vector $\boldsymbol{X}$ has a distribution governed by an unknown set of parameters $(\xi, \vartheta)$ where $\vartheta$ is real valued but $\xi$ may be vector valued or of arbitrary form. $\vartheta$ is the parameter of interest and often it is possible to reparametrize a given problem to fit this format.

A pivot for $\vartheta$ is a known function $\varphi(X, \vartheta)$ of the data vector $\mathbf{X}$ and the unknown parameter $\vartheta$ of interest with the following two properties

1. The cdf $H$ of the random variable $\varphi(X, \vartheta)$ is continuous and does not depend on any unknown parameters, i.e., does not depend on $(\xi, \vartheta)$.

2. For any fixed values of $\boldsymbol{X}$ the function $\varphi(\boldsymbol{X}, \vartheta)$ is strictly monotone increasing in $\vartheta$. Denote its inverse by $\varphi^{-1}(\cdot, \boldsymbol{X})$, i.e., $\varphi^{-1}(\varphi(\boldsymbol{X}, \vartheta), \boldsymbol{X})=\vartheta$ or $\varphi\left(\boldsymbol{X}, \varphi^{-1}(h, \boldsymbol{X})\right)=$ $h$.

The concept of a pivot is best examplified by its prime examples in the context of a normal random sample $X_{1}, \ldots, X_{n} \sim \mathscr{N}\left(\mu, \sigma^{2}\right)$, namely by $\sqrt{n}(\bar{X}-\mu) / s$ and $s^{2} / \sigma^{2}$, where $\bar{X}$ and $s^{2}$ are the sample mean and sample variance. These two pivots respectively have the known $t_{n-1}$ and $\chi_{n-1}^{2} /(n-1)$ distributions, independent of $\left(\mu, \sigma^{2}\right)$.

Returning to the generic pivot discussion, for a known $H$ and $p$-quantile $h_{p}$ of $H$ one can invert the following probability statement as shown

$$
\begin{aligned}
p=H\left(h_{p}\right) & =P\left(\varphi(\boldsymbol{X}, \vartheta) \leq h_{p}\right)=P\left(\varphi^{-1}(\varphi(\boldsymbol{X}, \vartheta), \boldsymbol{X})\right. \\
& \left.\leq \varphi^{-1}\left(h_{p}, \boldsymbol{X}\right)\right)=P\left(\vartheta \leq \varphi^{-1}\left(h_{p}, \boldsymbol{X}\right)\right)
\end{aligned}
$$

Thus $\varphi^{-1}\left(h_{p}, \boldsymbol{X}\right)$ serves as a $100 p \%$ upper confidence bound for the unknown parameter $\vartheta$. Finding $\varphi^{-1}\left(h_{p}, \boldsymbol{X}\right)$ just means solving $\varphi(\boldsymbol{X}, \vartheta)=h_{p}$ for $\vartheta=\varphi^{-1}\left(h_{p}, \boldsymbol{X}\right)$.

We may also allow $\varphi(\boldsymbol{X}, \vartheta)$ to be strictly decreasing in $\vartheta$ instead. That would only result in some reversed inequalities above, i.e., $100 p \%$ upper bounds would become $100 p \%$ lower bounds.

The distribution $H$ is either known explicitly (as in the normal example case above) and $p$-quantiles $h_{p}$ can be computed or $H$ and its quantiles can be approximated empirically for some conveniently chosen value of $(\xi, \vartheta)$ by simulating the data vector $\boldsymbol{X}$ and thus $\varphi(\boldsymbol{X}, \vartheta)$ a large number of times from the distribution characterized by the chosen $(\xi, \vartheta)$. By assumption the distribution of $\varphi(\boldsymbol{X}, \vartheta)$ will not depend on the conveniently chosen value $(\xi, \vartheta)$. This will all become less abstract in the examples presented below or should be familiar from the normal example presented above.

Returning from the generic situation, recall that for a Weibull random sample $\mathbf{X}=\left(X_{1}, \ldots, X_{n}\right)$ we have $Y_{i}=$ $\log \left(X_{i}\right) \sim G((y-u) / b)$ with $b=1 / \beta$ and $u=\log (\alpha)$. Then $Z_{i}=\left(Y_{i}-u\right) / b \sim G(z)=1-\exp (-\exp (z))$, which is the standard Gumbel distribution. In its standard form it does not depend on unknown parameters. This is seen as follows:

$$
\begin{aligned}
P\left(Z_{i} \leq z\right) & =P\left(\left(Y_{i}-u\right) / b \leq z\right)=P\left(Y_{i} \leq u+b z\right) \\
& =G(([u+b z]-u) / b)=G(z) .
\end{aligned}
$$

It is this known distribution of $\mathbf{Z}=\left(Z_{1}, \ldots, Z_{n}\right)$ that is instrumental in knowing (via simulation) the distribution of the four pivots that we discuss below. There we utilize the representation $Y_{i}=u+b Z_{i}$ or $\mathbf{Y}=u+b \mathbf{Z}$ in vector form.

\section{Pivot for the Scale Parameter $b$}

As natural pivot for the scale parameter $\vartheta=b$ we take

$$
W_{1}=\frac{\hat{b}(\mathbf{Y})}{b}=\frac{\hat{b}(u+b \mathbf{Z})}{b}=\frac{b \hat{b}(\mathbf{Z})}{b}=\hat{b}(\mathbf{Z}) .
$$

The right side, being a function of $\mathbf{Z}$ alone, has a distribution that does not involve unknown parameters and $W_{1}=$ $\hat{b}(\mathbf{Y}) / b$ is strictly monotone in $b$.

How do we obtain the distribution of $\hat{b}(\mathbf{Z})$ ? An analytical approach does not seem possible. The approach followed here is that presented in Bain, 1978, Bain and Engelhardt, 1991 and originally in Thoman et al., 1969 and Thoman et al., 1970, which provided tables for this distribution (and for those of the other pivots discussed here) based on $N_{\text {sim }}$ simulated values of $\hat{b}(\mathbf{Z})$ (and $\hat{u}(\mathbf{Z})$ ), where $N_{\text {sim }}=20000$ for $n=5, N_{\text {sim }}=10000$ for $n=$ $6,8,10,15,20,30,40,50,75$, and $N_{\text {sim }}=6000$ for $n=100$.

In these simulations one simply generates samples $\mathbf{Z}^{\star}=\left(Z_{1}, \ldots, Z_{n}\right) \sim G(z)$ and finds $\hat{b}\left(\mathbf{Z}^{\star}\right)$ (and $\hat{u}\left(\mathbf{Z}^{\star}\right)$ for the other pivots discussed later) for each such sample $\mathbf{Z}^{\star}$. By simulating this process $N_{\text {sim }}=10000$ times we obtain $\hat{b}\left(\mathbf{Z}_{1}^{\star}\right), \ldots, \hat{b}\left(\mathbf{Z}_{N_{\text {sim }}}^{\star}\right)$. The empirical distribution function of these simulated estimates $\hat{b}\left(\mathbf{Z}_{i}^{\star}\right)$, denoted by $\hat{H}_{1}(w)$, provides a fairly reasonable estimate of the sampling distribution $H_{1}(w)$ of $\hat{b}(\mathbf{Z})$ and thus also of the pivot distribution of $W_{1}=\hat{b}(\mathbf{Y}) / b$. From this simulated distribution we can estimate any $\gamma$-quantile of $H_{1}(w)$ to any practical accuracy, provided $N_{\text {sim }}$ is sufficiently large. Values of $\gamma$ closer to 0 or 
Figure 6 - Interpolation \& Extrapolation for $A^{2}(1+.2 / \sqrt{n})$ and $W^{2}(1+.2 / \sqrt{n})$

(a)

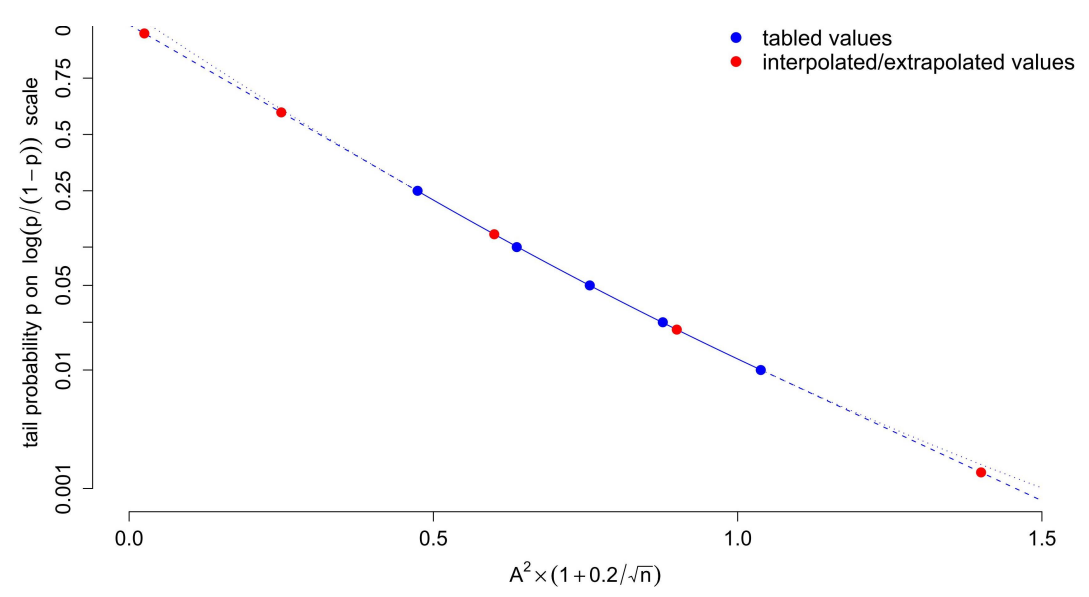

(b)

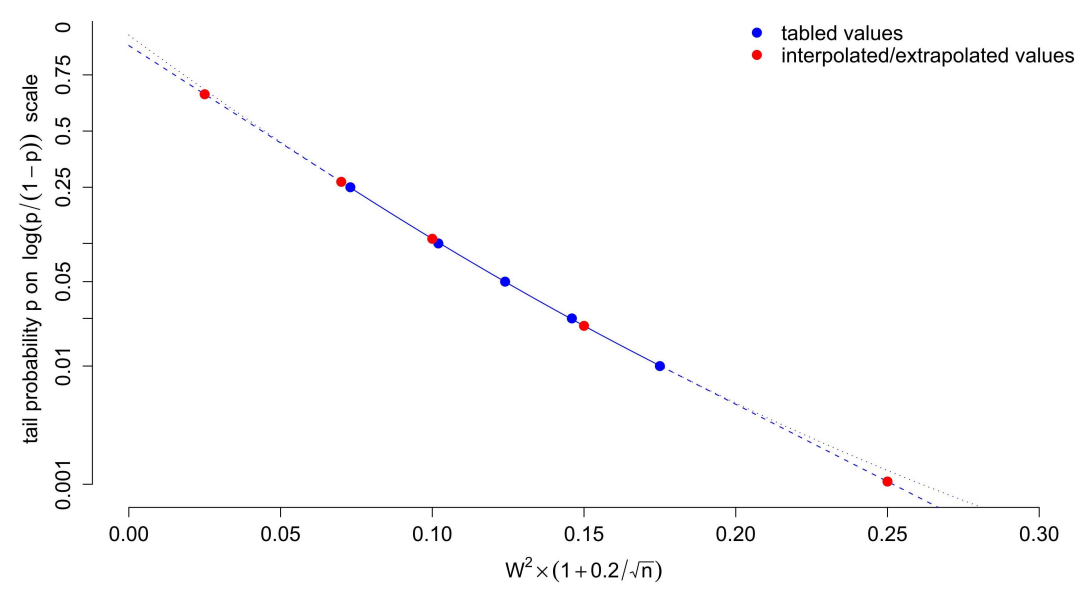

1 require higher $N_{\text {sim }}$. For $.005 \leq \gamma \leq .995$ a simulation level of $N_{\text {sim }}=10000$ should be quite adequate.

If we denote the $\gamma$-quantile of $H_{1}(w)$ by $\eta_{1}(\gamma)$, i.e.,

$$
\gamma=H_{1}\left(\eta_{1}(\gamma)\right)=P\left(\hat{b}(\mathbf{Y}) / b \leq \eta_{1}(\gamma)\right)=P\left(\hat{b}(\mathbf{Y}) / \eta_{1}(\gamma) \leq b\right)
$$

we see that $\hat{b}(\mathbf{Y}) / \eta_{1}(\gamma)$ can be viewed as a $100 \gamma \%$ lower bound to the unknown parameter $b$. We do not know $\eta_{1}(\gamma)$ but we can estimate it by the corresponding quantile $\hat{\eta}_{1}(\gamma)$ of the simulated distribution $\hat{H}_{1}(w)$ which serves as proxy for $H_{1}(w)$. We then use $\hat{b}(\mathbf{Y}) / \hat{\eta}_{1}(\gamma)$ as an approximate $100 \gamma \%$ lower bound to the unknown parameter $b$. For large $N_{\text {sim }}$, say $N_{\text {sim }}=10000$, this approximation is practically quite adequate.

We note here that a $100 \gamma \%$ lower bound can be viewed as a $100(1-\gamma) \%$ upper bound, because $1-\gamma$ is the chance of the lower bound falling on the wrong side of its target, namely above. The chance for equality is zero since the distribution of $\hat{b}(\mathbf{Y})$ is continuous (no proof of that is given here). To get $100 \gamma \%$ upper bounds one simply constructs $100(1-\gamma) \%$ lower bounds by the above method. Similar comments apply to the pivots obtained below, where we only give one-sided bounds (lower or upper) in each case.

Based on the relationship $b=1 / \beta$ the respective $100 \gamma \%$ approximate lower and upper confidence bounds for the Weibull shape parameter would be

$\frac{\hat{\eta}_{1}(1-\gamma)}{\hat{b}(\mathbf{Y})}=\hat{\eta}_{1}(1-\gamma) \times \hat{\beta}(\mathbf{X}) \quad$ and $\quad \frac{\hat{\eta}_{1}(\gamma)}{\hat{b}(\mathbf{Y})}=\hat{\eta}_{1}(\gamma) \times \hat{\beta}(\mathbf{X})$ and an approximate $100 \gamma \%$ confidence interval for $\beta$ would be

$$
\left[\hat{\eta}_{1}((1-\gamma) / 2) \times \hat{\beta}(\mathbf{X}), \hat{\eta}_{1}((1+\gamma) / 2) \times \hat{\beta}(\mathbf{X})\right]
$$

since $(1+\gamma) / 2=1-(1-\gamma) / 2$. Here $\mathbf{X}=\left(X_{1}, \ldots, X_{n}\right)$ is the untransformed Weibull sample. 


\section{Pivot for the Location Parameter $u$}

For the location parameter $\vartheta=u$ we have the following pivot

$$
W_{2}=\frac{\hat{u}(\mathbf{Y})-u}{\hat{b}(\mathbf{Y})}=\frac{\hat{u}(u+b \mathbf{Z})-u}{\hat{b}(u+b \mathbf{Z})}=\frac{u+b \hat{u}(\mathbf{Z})-u}{b \hat{b}(\mathbf{Z})}=\frac{\hat{u}(\mathbf{Z})}{\hat{b}(\mathbf{Z})} .
$$

It has a distribution that does not depend on any unknown parameters, since it only depends on the known distribution of $\mathbf{Z}$. Furthermore $W_{2}$ is strictly decreasing in $u$. Thus $W_{2}$ is a pivot with respect to $u$. Denote this pivot distribution of $W_{2}$ by $H_{2}(w)$ and its $\gamma$-quantile by $\eta_{2}(\gamma)$. As before this pivot distribution and its quantiles can be approximated sufficiently well by simulating $\hat{u}\left(\mathbf{Z}^{\star}\right) / \hat{b}\left(\mathbf{Z}^{\star}\right)$ a sufficient number $N_{\text {sim }}$ times and using the empirical cdf $\hat{H}_{2}(w)$ of the $\hat{u}\left(\mathbf{Z}_{i}^{\star}\right) / \hat{b}\left(\mathbf{Z}_{i}^{\star}\right)$ as proxy for $H_{2}(w)$.

As in the previous pivot case we can exploit this pivot distribution as follows

$\gamma=H_{2}\left(\eta_{2}(\gamma)\right)=P\left(\frac{\hat{u}(\mathbf{Y})-u}{\hat{b}(\mathbf{Y})} \leq \eta_{2}(\gamma)\right)=P\left(\hat{u}(\mathbf{Y})-\hat{b}(\mathbf{Y}) \eta_{2}(\gamma) \leq u\right)$

and thus we can view $\hat{u}(\mathbf{Y})-\hat{b}(\mathbf{Y}) \eta_{2}(\gamma)$ as a $100 \gamma \%$ lower bound for the unknown parameter $u$. Using the $\gamma$-quantile $\hat{\eta}_{2}(\gamma)$ obtained from the empirical cdf $\hat{H}_{2}(w)$ we then treat $\hat{u}(\mathbf{Y})-\hat{b}(\mathbf{Y}) \hat{\eta}_{2}(\gamma)$ as an approximate $100 \gamma \%$ lower bound for the unknown parameter $u$.

Based on the relation $u=\log (\alpha)$ this translates into an approximate $100 \gamma \%$ lower bound

$$
\begin{aligned}
\exp \left(\hat{u}(\mathbf{Y})-\hat{b}(\mathbf{Y}) \hat{\eta}_{2}(\gamma)\right) & =\exp \left(\log (\hat{\alpha}(\mathbf{X}))-\hat{\eta}_{2}(\gamma) / \hat{\beta}(\mathbf{X})\right) \\
& =\hat{\alpha}(\mathbf{X}) \exp \left(-\hat{\eta}_{2}(\gamma) / \hat{\beta}(\mathbf{X})\right) \quad \text { for } \alpha
\end{aligned}
$$

Upper bounds and intervals for $u$ or $\alpha$ are handled as in the previous situation for $b$ or $\beta$.

\section{Pivot for the $p$-quantile $y_{p}$}

With respect to the $p$-quantile $\vartheta=y_{p}=u+b \log (-\log (1-$ $p))=u+b w_{p}$ of the $Y$ distribution the natural pivot is

$$
\begin{aligned}
W_{p}=\frac{\hat{y}_{p}(\mathbf{Y})-y_{p}}{\hat{b}(\mathbf{Y})} & =\frac{\hat{u}(\mathbf{Y})+\hat{b}(\mathbf{Y}) w_{p}-\left(u+b w_{p}\right)}{\hat{b}(\mathbf{Y})} \\
& =\frac{\hat{u}(u+b \mathbf{Z})+\hat{b}(u+b \mathbf{Z}) w_{p}-\left(u+b w_{p}\right)}{\hat{b}(u+b \mathbf{Z})} \\
& =\frac{u+b \hat{u}(\mathbf{Z})+b \hat{b}(\mathbf{Z}) w_{p}-\left(u+b w_{p}\right)}{b \hat{b}(\mathbf{Z})} \\
& =\frac{\hat{u}(\mathbf{Z})+(\hat{b}(\mathbf{Z})-1) w_{p}}{\hat{b}(\mathbf{Z})} .
\end{aligned}
$$

Again its distribution only depends on the known distribution of $\mathbf{Z}$ and not on the unknown parameters $u$ and $b$ and the pivot $W_{p}$ is a strictly decreasing function of $y_{p}$. Denote this pivot distribution function by $H_{p}(w)$ and its $\gamma$-quantile by $\eta_{p}(\gamma)$. This pivot distribution and its quantiles can be approximated sufficiently well by simulating $\left\{\hat{u}(\mathbf{Z})+(\hat{b}(\mathbf{Z})-1) w_{p}\right\} / \hat{b}(\mathbf{Z})$ a sufficient number $N_{\text {sim }}$ times. Denote the empirical cdf of such simulated values by $\hat{H}_{p}(w)$ and the corresponding $\gamma$-quantiles by $\hat{\eta}_{p}(\gamma)$.

As before we proceed with

$$
\begin{aligned}
\gamma=H_{p}\left(\eta_{p}(\gamma)\right) & =P\left(\frac{\hat{y}_{p}(\mathbf{Y})-y_{p}}{\hat{b}(\mathbf{Y})} \leq \eta_{p}(\gamma)\right) \\
& =P\left(\hat{y}_{p}(\mathbf{Y})-\eta_{p}(\gamma) \hat{b}(\mathbf{Y}) \leq y_{p}\right)
\end{aligned}
$$

and thus we can treat $\hat{y}_{p}(\mathbf{Y})-\eta_{p}(\gamma) \hat{b}(\mathbf{Y})$ as a $100 \gamma \%$ lower bound for $y_{p}$. Again we can treat $\hat{y}_{p}(\mathbf{Y})-\hat{\eta}_{p}(\gamma) \hat{b}(\mathbf{Y})$ as an approximate $100 \gamma \%$ lower bound for $y_{p}$.

Since

$$
\begin{aligned}
\hat{y}_{p}(\mathbf{Y})-\eta_{p}(\gamma) \hat{b}(\mathbf{Y}) & =\hat{u}(\mathbf{Y})+w_{p} \hat{b}(\mathbf{Y})-\eta_{p}(\gamma) \hat{b}(\mathbf{Y}) \\
& =\hat{u}(\mathbf{Y})-k_{p}(\gamma) \hat{b}(\mathbf{Y})
\end{aligned}
$$

with $k_{p}(\gamma)=\eta_{p}(\gamma)-w_{p}$, we could have obtained the same lower bound by the following argument that does not use a direct pivot, namely

$$
\begin{aligned}
\gamma & =P\left(\hat{u}(\mathbf{Y})-k_{p}(\gamma) \hat{b}(\mathbf{Y}) \leq y_{p}\right) \\
& =P\left(\hat{u}(\mathbf{Y})-k_{p}(\gamma) \hat{b}(\mathbf{Y}) \leq u+b w_{p}\right) \\
& =P\left(\hat{u}(\mathbf{Y})-u-k_{p}(\gamma) \hat{b}(\mathbf{Y}) \leq b w_{p}\right) \\
& =P\left(\frac{\hat{u}(\mathbf{Y})-u}{b}-k_{p}(\gamma) \frac{\hat{b}(\mathbf{Y})}{b} \leq w_{p}\right) \\
& =P\left(\hat{u}(\mathbf{Z})-k_{p}(\gamma) \hat{b}(\mathbf{Z}) \leq w_{p}\right) \\
& =P\left(\frac{\hat{u}(\mathbf{Z})-w_{p}}{\hat{b}(\mathbf{Z})} \leq k_{p}(\gamma)\right)
\end{aligned}
$$

and we see that $k_{p}(\gamma)$ can be taken as the $\gamma$-quantile of the distribution of $\left(\hat{u}(\mathbf{Z})-w_{p}\right) / \hat{b}(Z)$.

This distribution can be estimated by the empirical cdf of $N_{\text {sim }}$ simulated values $\left(\hat{u}\left(\mathbf{Z}_{i}^{\star}\right)-w_{p}\right) / \hat{b}\left(\mathbf{Z}_{i}^{\star}\right), i=1, \ldots, N_{\text {sim }}$ and its $\gamma$-quantile $\hat{k}_{p}(\gamma)$ serves as a good approximation to $k_{p}(\gamma)$.

It is easily seen that this produces the same quantile lower bound as before. However, in this approach one sees one further detail, namely that $h(p)=-k_{p}(\gamma)$ is strictly increasing in $p^{2}$, since $w_{p}$ is strictly increasing in $p$.

\footnotetext{
${ }^{2}$ Suppose $p_{1}<p_{2}$ and $h\left(p_{1}\right) \geq h\left(p_{2}\right)$ with $\gamma=P\left(\hat{u}(\mathbf{Z})+h\left(p_{1}\right) \hat{b}(\mathbf{Z}) \leq w_{p_{1}}\right)$ and $\gamma=P\left(\hat{u}(\mathbf{Z})+h\left(p_{2}\right) \hat{b}(\mathbf{Z}) \leq w_{p_{2}}\right)=P\left(\hat{u}(\mathbf{Z})+h\left(p_{1}\right) \hat{b}(\mathbf{Z}) \leq w_{p_{1}}+\left(w_{p_{2}}-w_{p_{1}}\right)+\right.$ $\left.\left(h\left(p_{1}\right)-h\left(p_{2}\right)\right) \hat{b}(\mathbf{Z})\right) \geq P\left(\hat{u}(\mathbf{Z})+h\left(p_{1}\right) \hat{b}(\mathbf{Z}) \leq w_{p_{1}}+\left(w_{p_{2}}-w_{p_{1}}\right)\right)>\gamma$ (i.e., $\gamma>\gamma$, a contradiction) since $P\left(w_{p_{1}}<\hat{u}(\mathbf{Z})+h\left(p_{1}\right) \hat{b}(\mathbf{Z}) \leq w_{p_{1}}+\left(w_{p_{2}}-w_{p_{1}}\right)\right)>0$. A thorough argument would show that $\hat{b}(\mathbf{z})$ and thus $\hat{u}(\mathbf{z})$ are continuous functions of $\mathbf{z}=\left(z_{1}, \ldots, z_{n}\right)$ and since there is positive probability in any neighborhood of any $\mathbf{z} \in R$ there is positive probability in any neighborhood of $(\hat{u}(\mathbf{z}), \hat{b}(\mathbf{z}))$.
} 
Of course it makes intuitive sense that quantile lower bounds should be increasing in $p$ since its target $p$ quantiles are increasing in $p$. This strictly increasing property allows us to immediately construct upper confidence bounds for left tail probabilities as is shown in the next section.

Since $x_{p}=\exp \left(y_{p}\right)$ is the corresponding $p$-quantile of the Weibull distribution we can view

$$
\begin{aligned}
\exp \left(\hat{y}_{p}(\mathbf{Y})-\hat{\eta}_{p}(\gamma) \hat{b}(\mathbf{Y})\right) & =\hat{\alpha}(\mathbf{X}) \exp \left(\left(w_{p}-\hat{\eta}_{p}(\gamma)\right) / \hat{\beta}(\mathbf{X})\right) \\
& =\hat{\alpha}(\mathbf{X}) \exp \left(-\hat{k}_{p}(\gamma) / \hat{\beta}(\mathbf{X})\right)
\end{aligned}
$$

as an approximate $100 \gamma \%$ lower bound for $x_{p}=\exp (u+$ $\left.b w_{p}\right)=\alpha(-\log (1-p))^{1 / \beta}$.

Since $\alpha$ is the (1-exp(-1))-quantile of the Weibull distribution, lower bounds for it can be seen as a special case of quantile lower bounds. Indeed, this particular quantile lower bound coincides with the one given previously.

\section{Upper Confidence Bounds for the Tail Probability} $p(y)=P(Y \leq y)$

As far as an appropriate pivot for $p(y)=P(Y \leq y)$ is concerned, the situation here is not as straightforward as in the previous three cases. Clearly

$$
\hat{p}(y)=G\left(\frac{y-\hat{u}(\mathbf{Y})}{\hat{b}(\mathbf{Y})}\right)
$$

is the natural estimate (mle) of

$$
p(y)=P(Y \leq y)=G\left(\frac{y-u}{b}\right)
$$

and one easily sees that the distribution function $H$ of this estimate depends on $u$ and $b$ only through $p(y)$, namely

$$
\begin{aligned}
\hat{p}(y) & =G\left(\frac{y-\hat{u}(\mathbf{Y})}{\hat{b}(\mathbf{Y})}\right)=G\left(\frac{(y-u) / b-(\hat{u}(\mathbf{Y})-u) / b}{\hat{b}(\mathbf{Y}) / b}\right) \\
& =G\left(\frac{G^{-1}(p(y))-\hat{u}(\mathbf{Z})}{\hat{b}(\mathbf{Z})}\right) \sim H_{p(y)} .
\end{aligned}
$$

Thus by the probability integral transform it follows that

$$
W_{p(y)}=H_{p(y)}(\hat{p}(y)) \sim U(0,1)
$$

i.e., $W_{p(y)}$ is a true pivot, contrary to what is stated in Bain, 1978 and Bain and Engelhardt, 1991.

Rather than using this pivot we will go a more direct route as was indicated by the strictly increasing property of $h(p)=h_{\gamma}(p)$ in the previous section. Denote by $h^{-1}(\cdot)$ the inverse function to $h(\cdot)$. We then have

$$
\begin{aligned}
\gamma & =P\left(\hat{u}(\mathbf{Y})+h(p) \hat{b}(\mathbf{Y}) \leq y_{p}\right)=P\left(h(p) \leq\left(y_{p}-\hat{u}(\mathbf{Y})\right) / \hat{b}(\mathbf{Y})\right) \\
& =P\left(p \leq h^{-1}\left(\left(y_{p}-\hat{u}(\mathbf{Y})\right) / \hat{b}(\mathbf{Y})\right)\right)
\end{aligned}
$$

for any $p \in(0,1)$. If we parameterize such $p$ via $p(y)=$ $P(Y \leq y)=G((y-u) / b)$ we have $y_{p(y)}=y$ and thus also

$$
\gamma=P\left(p(y) \leq h^{-1}((y-\hat{u}(\mathbf{Y})) / \hat{b}(\mathbf{Y}))\right)
$$

for any $y \in R$ and $u \in R$ and $b>0$. Hence $\hat{p}_{U}(y)=$ $h^{-1}((y-\hat{u}(\mathbf{Y})) / \hat{b}(\mathbf{Y}))$ can be viewed as $100 \gamma \%$ upper confidence bound for $p(y)$ for any given threshold $y$.

The only remaining issue is the computation of such bounds. Does it require the inversion of $h$ and the concomitant calculations of many $h(p)=-k(p)$ for the iterative convergence of such an inversion? It turns out that there is a direct path just as we had it in the previous three confidence bound situations.

Note that $h^{-1}(x)$ solves $-k_{p}=x$ for $p$. We claim that $h^{-1}(x)$ is the $\gamma$-quantile of the $G(\hat{u}(\mathbf{Z})+x \hat{b}(\mathbf{Z}))$ distribution which we can simulate by calculating as before $\hat{u}(\mathbf{Z})$ and $\hat{b}(\mathbf{Z})$ a large number $N_{\text {sim }}$ times. The above claim concerning $h^{-1}(x)$ is seen as follows. If for any $x=h(p)$ we have

$$
\begin{aligned}
P\left(G(\hat{u}(\mathbf{Z})+x \hat{b}(\mathbf{Z})) \leq h^{-1}(x)\right) & =P(G(\hat{u}(\mathbf{Z})+h(p) \hat{b}(\mathbf{Z})) \leq p) \\
& =P\left(\hat{u}(\mathbf{Z})+h(p) \hat{b}(\mathbf{Z}) \leq w_{p}\right) \\
& =P\left(\hat{u}(\mathbf{Z})-k_{\gamma}(p) \hat{b}(\mathbf{Z}) \leq w_{p}\right)=\gamma,
\end{aligned}
$$

as seen in the previous section. Thus $h^{-1}(x)$ is the $\gamma$ quantile of the $G(\hat{u}(\mathbf{Z})+x \hat{b}(\mathbf{Z}))$ distribution.

If we observe $\mathbf{Y}=\mathbf{y}$ and obtain $\hat{u}(\mathbf{y})$ and $\hat{b}(\mathbf{y})$ as our maximum likelihood estimates for $u$ and $b$ we get our $100 \gamma \%$ upper bound for $p(y)=G((y-u) / b)$ as follows: For the fixed value of $x=(y-\hat{u}(\mathbf{y})) / \hat{b}(\mathbf{y})=G^{-1}(\hat{p}(y))$ simulate the $G(\hat{u}(\mathbf{Z})+x \hat{b}(\mathbf{Z}))$ distribution (with sufficiently high $N_{\text {sim }}$ ) and calculate the $\gamma$-quantile of this distribution as the desired approximate $100 \gamma \%$ upper bound for $p(y)=P(Y \leq$ $y)=G((y-u) / b)$.

\section{Tabulation of Confidence Quantiles $\eta(\gamma)$}

For the pivots for $b, u$ and $y_{p}$ it is possible to carry out simulations once and for all for a desired set of confidence levels $\gamma$, sample sizes $n$ and choices of $p$, and tabulate the required confidence quantiles $\hat{\eta}_{1}(\gamma), \hat{\eta}_{2}(\gamma)$, and $\hat{\eta}_{p}(\gamma)$. This has essentially been done (with $\sqrt{n}$ scaling modifications) and such tables are given in Bain, 1978, Bain and Engelhardt, 1991, Thoman et al., 1969 and Thoman et al., 1970. Similar tables for bounds on $p(y)$ are not quite possible since the appropriate bounds depend on the observed value of $\hat{p}(y)$, which varies from sample to sample. Instead Bain, 1978, Bain and Engelhardt, 1991 and Thoman et al., 1970 tabulate confidence bounds for $p(y)$ for a reasonably fine grid of values for $\hat{p}(y)$, which can then serve for interpolation purposes with the actually observed value of $\hat{p}(y)$.

It should be quite clear that all this requires extensive tabulation. The use of these tables is not easy. Table 4 in Bain, 1978 does not have a consistent format and using these tables would require delving deeply into the text 
for each new use, unless one does this kind of calculation all the time. In fact, in the second edition Bain and Engelhardt, 1991 Table 4 has been greatly reduced to just cover the confidence factors dealing with the location parameter $u$, and it now leaves out the confidence factors for general $p$-quantiles. For the $p$-quantiles one is referred to the same interpolation scheme that is needed when getting confidence bounds for $p(y)$, using Table 7 in Bain and Engelhardt, 1991. The example that they present (page 248) would have benefitted by showing some intermediate steps in the interpolation process. They point out that the resulting confidence bound for $x_{p}$ is slightly different (14.03) from that obtained using the confidence quantiles of the original Table 4, namely 13.92. They attribute the difference to round-off errors or other discrepancies. Among the latter one may consider that possibly different simulations were involved.

Further, note that some entries in the tables given in Bain, 1978 seem to have typos. Presumably they were transcribed by hand from computer output, just as the book (and its second edition) itself is typed and not typeset. We just give a few examples. In Bain, 1978 Table 4A, p.235, bottom row, the second entry from the right should be 3.625 instead of 3.262. This discrepancy shows up clearly when plotting the row values against $\log (p /(1-p))$, see a similar plot for a later example. In Table 3A, p.222, row 3 column 5 shows a double minus sign (still present the second edition Bain and Engelhardt, 1991). In comparing the values of these tables with our own simulation of pivot distribution quantiles, just to validate our simulation for $n=40$, we encountered an apparent error in Table 4A, p. 235 with last column entry of 4.826 . Plotting $\log (p /(1-p))$ against the corresponding row value ( $\gamma$-quantiles) one clearly sees a change in pattern, see the top plot in Figure 8. We suspect that the whole last column was calculated for $p=.96$ instead of the indicated $p=.98$. The bottom plot shows our simulated values for these quantiles as solid dots with the previous points (circles) superimposed. Both plots were produced by test 40() .

The agreement is good for the first 8 points. Our simulated $\gamma$-quantile was 5.725 (corresponding to the 4.826 above) and it fits quite smoothly into the pattern of the previous 8 points. Given that this was the only case chosen for comparison it leaves some concern in fully trusting these tables. However, this example also shows that the great majority of tabled values are valid.

\section{The R Function WeibullPivots}

Rather than using these tables we will resort to direct simulations ourselves since computing speed and availability have advanced sufficiently over what was common prior to 1978. This is implemented in the function
Weibullpivots.

The call

system.time (WeibullPivots (

Nsim=10000, $n=10, r=10$, graphics=F))

gave an elapsed time of 15.28 seconds. Here the default sample size $n=10$ was used and $r=10$ (also default) indicates that the 10 lowest sample values are given and used, i.e., in this case the full sample. Also, an internally generated Weibull data set was used, since the default in the call to WeibullPivots is weib. sample=NULL. For sample sizes $n=100$ with $r=100$ and $n=$ 1000 with $r=1000$ the corresponding calls resulted in elapsed times of 17.78 and 56.59 seconds, respectively. These three computing times suggest strong linear behavior in $n$ as is illustrated in Figure 9, produced by WeibullPivot.timing.plot (). The intercept 14.24 and slope of .04229 given here are fairly consistent with the intercept .001402 and slope of $5.072 \times 10^{-6}$ given in Figure 3 . The latter give the calculation time of a single set of mle's while in the former case we calculate $N_{\text {sim }}=10000 \mathrm{such}$ mle's, i.e., the previous slope and intercept for a single mle calculation need to be scaled up by the factor 10000 .

For all the previously discussed confidence bounds, be they upper or lower bounds for their respective targets, all that is needed is the set of $\left(\hat{u}\left(\mathbf{z}_{i}\right), \hat{b}\left(\mathbf{z}_{i}\right)\right)$ for $i=1, \ldots, N_{\text {sim }}$. Thus we can construct confidence bounds and intervals for $u$ and $b$, for $y_{p}$ for any collection of values $p$, and for $p(y)$ and $1-p(y)$ for any collection of threshold values $y$ and we can do this for any set of confidence levels that make sense for the simulated distributions, i.e., we don't have to run the simulations over and over for each target parameter, confidence level, $p$ or $y$, unless one wants independent simulations for some reason.

Proper use of this function only requires understanding the calling arguments, purpose, and output of this function, and the time to run the simulations. The time for running the simulation should easily beat the time spent in dealing with tabulated confidence quantiles in order to get desired confidence bounds, especially since WeibullPivots does such calculations all at once for a broad spectrum of $y_{p}$ and $p(y)$ and several confidence levels without greatly impacting the computing time. Furthermore, WeibullP ivots does all this not only for full samples but also for type II censored samples, for which appropriate confidence factors are available only sparsely in tables.

We now explain input and output of the function WeibullPivots. The calling sequence with all arguments given with their default values is as follows:

WeibullPivots (weib. sample=NULL, alpha $=10000$, bet $a=1.5, n=10, r=10$, 


\section{Nsim=1000, threshold=NULL, graphics $=\mathrm{T}$ )}

Here Nsim $=N_{\text {sim }}$ has default value 1000 which is appropriate when trying to get a feel for the function for any particular data set. The sample size is input as $\mathrm{n}=n$ and $r=r$ indicates the number of smallest sample values available for analysis. When $r<n$ we are dealing with a type II censored data set where observation stops as soon as the smallest $r$ lifetimes have been observed.

We need $r>1$ and at least two distinct observations among $X_{(1)}, \ldots, X_{(r)}$ in order to estimate any spread in the data. The available sample values $X_{1}, \ldots, X_{r}$ (not necessarily ordered) are given as vector input to weib. sample. When weib.sample=NULL (the default), an internal data set is generated as input sample from $\mathbb{W}(\alpha, \beta)$ with $\alpha=$ alpha $=10000$ (default) and $\beta=$ beta $=1.5$ (default), either by using the full sample $X_{1}, \ldots, X_{n}$ or a type II censored sample $X_{1}, \ldots, X_{r}$ when $r<n$ is specified. The input thresh (= NULL by default) is a vector of thresholds $y$ for which we desire upper confidence bounds for $p(y)$. The input graphics (default $\mathrm{T}$ ) indicates whether graphical output is desired.

Confidence levels $\gamma$ are set internally as $.005, .01, .025, .05, .10, .02, .8, .9, .95, .975, .99, .995$ and these levels indicate the coverage probability for the individual one-sided bounds. A .025 lower bound is reported as a .975 upper bound, and a pair of .975 lower and upper bounds constitute a 95\% confidence interval. The values of $p$ for which confidence bounds or intervals for $x_{p}$ are provided are also set internally as $.001, .005, .01, .025, .05, .1,(.1), .9, .95, .975, .99, .995, .999$.

The output from WeibullPivots is a list with components:

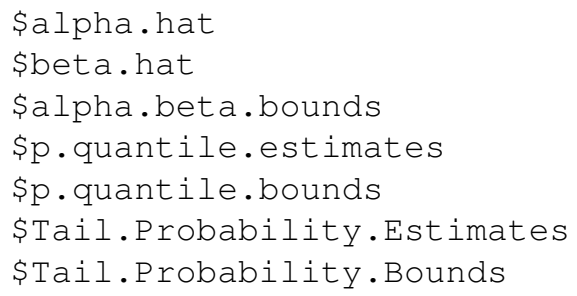

The structure and meaning of these components will become clear from the example output given in Outputs 1, 2 and 3.

These outputs were produced with

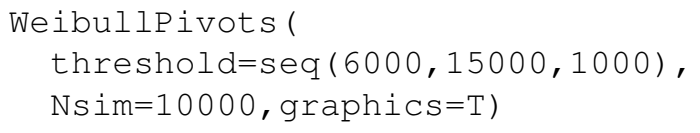

Since we entered graphics $=\mathrm{T}$ as argument we also got two pieces of graphical output. The first gives the two intrinsic pivot distributions of $\hat{u} / \hat{b}$ and $\hat{b}$ in Figure 10. The second gives a Weibull plot of the generated sample with a variety of information and with several types of confidence bounds, see Figure 11. The legend in the upper left gives the mle's of $\alpha, \beta$ (agreeing with the output above), and the mean $\mu=\alpha \Gamma(1+1 / \beta)$ together with $95 \%$ confidence intervals, based on respective normal approximation theory for the mle's. The legend in the lower right explains the red fitted line (representing the mle fit) and the various point-wise confidence bound curves, giving 95\% confidence intervals (blue dashed curves) for $p$-quantiles $x_{p}$ for any $p$ on the ordinate and 95\% confidence intervals (green dot-dashed line) for $p(y)$ for any $y$ on the abscissa. Both of these interval types use normal approximations from large sample mle theory. Unfortunately these two types of bounds are not dual to each other, i.e., don't coincide or to say it differently, one is not the inverse to the other.

A third type of bound is presented in the orange curve which simultaneously provides $95 \%$ confidence intervals for $x_{p}$ and $p(x)$, depending on the direction in which the curves are used. We either read sideways from $p$ and down from the curve (at that $p$ level) to get upper and lower bounds for $x_{p}$, or we read vertically up from an abscissa value $x$ to read off upper and lower bounds for $p(x)$ on the ordinate axis as we go from the respective curves at that $x$ value to the left. These latter bounds are also based on normal mle approximation theory and the approximation will naturally suffer for small sample sizes. However, the principle behind these bounds is a unifying one in that the same curve is used for quantile and tail probability bounds. If instead of using the approximating normal distribution one uses the parametric bootstrap approach Scholz, 1994(simulating samples from an estimated Weibull distribution) the unifying principle reduces to the pivot simulation approach, i.e., is basically exact except for the simulation aspect $N_{\text {sim }}<\infty$.

The curves representing the latter (pivots with simulated distributions) are the solid black lines connecting the solid black dots which represent the $x_{p} 95 \%$ confidence intervals (using the $97.5 \%$ lower and upper bounds to $x_{p}$ given in our output example above. Also seen on these curves are solid red dots that correspond to the abscissa values $x=6000$,(1000), 15000 and viewed vertically they represent $95 \%$ confidence intervals for $p(x)$. This illustrates that the same curves are used.

Figure 12 represents an extreme case where we have a sample of size $n=2$ and here another issue arises. Both of the first two types of bounds (blue and green) are no longer monotone in $p$ or $x$ respectively. This is the result of a poor normal approximation for these two approaches. Thus we could not (at least not generally) have taken either to take the role of serving both purposes, i.e., as providing bounds for $x_{p}$ and $p(x)$ simultaneously. However, the orange curve is still monotone and still serves that dual 
Output 1: Parameter estimates and parameter bounds

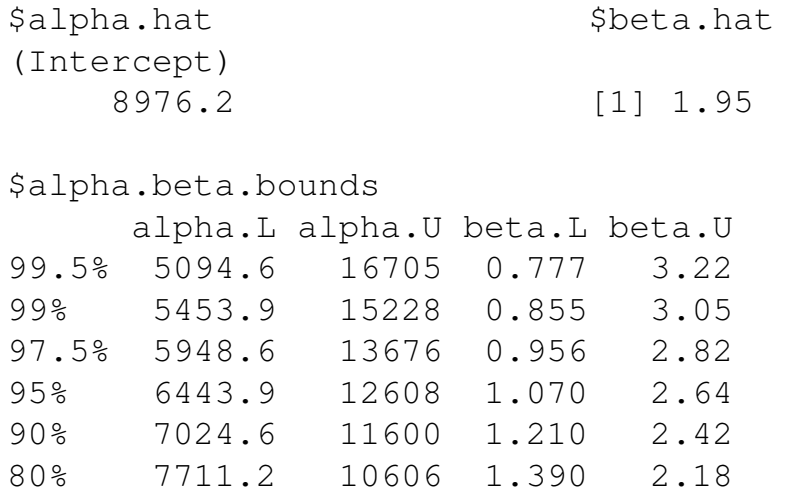

purpose, although its coverage probability properties are bound to be affected badly by the small sample size $n=2$. The pivot based curves are also strictly monotone and they have exact coverage probability, subject to the $N_{\text {sim }}<\infty$ limitation.

The supplied R function WeibullPivots is part of the collection of $\mathrm{R}$ code and data sets supplied in the file Weibull.txt available on the journal's web site. To make them available within an $\mathrm{R}$ session execute

source ("Weibull.txt")

assuming that Weibull.txt resides in the folder from which the $\mathrm{R}$ session was started. This collection contains all functions that were used in creating the plots in this tutorial and much more. These functions are documented internally. Also provided are some functions that allow usage not just for complete Weibull samples but also for type I censored Weibull data accompanied by covariates. For more information on this we refer to Scholz, 1996 (revised 2001).

\section{References}

Bain, L. J. (1978). Statistical analysis of reliability and lifetesting models. New York: Dekker.

Bain, L. J. \& Engelhardt, M. (1991). Statistical analysis of reliability and life-testing models, , theory and methods, second edition. New York: Dekker.

Castillo, E. (1988). Extreme value theory in engineering. Boston: Academic Press.

Coles, S. (2001). An introduction to statistical modeling of extremes. London: Springer.

Embrechts, P., Klüppelberg, C., \& Mikosch, T. (1997). Modelling extremal events. Berlin: Springer.

Gumbel, E. (1958). Statistics of extremes. New York: Columbia University Press.
Heller, R. A. (1985). The weibull distribution did not apply to its founder. In S. Eggwertz \& N. C. Lind (Eds.), Probabilistic methods in mechanics of solids and structures, the Weibull symposium. Berlin: Springer-Verlag.

R Core Team. (2015). R: a language and environment for statistical computing. R Foundation for Statistical Computing. Vienna, Austria. Retrieved from http: / / www. R-project.org/

Saunders, S. C. (1975). Birnbaum's contributions to reliability. In R. Barlow, J. Fussell, \& N. Singpurwalla (Eds.), Reliability and fault tree analysis, theoretical and applied aspects of system reliability and safety assessment. 33 South 17 Street, Philadelphia PA 19103: Society for Industrial and Applied Mathematics.

Scholz, F. W. (1994). On exactness of the parametric double bootstrap. Statistica Sinica, (4), 477-492.

Scholz, F. W. (1996 (revised 2001)). Maximum likelihood estimation for type I censored Weibull data including covariates (tech. rep. No. ISSTECH-96-022). Boeing Information and Support Services.

Stephens, M. A. (1986). Tests based on EDF statistics. In R. D’Agostino \& M. Stephens (Eds.), Goodness-of-fit techniques (pp. 97-193). New York: Dekker.

Thoman, D. R., Bain, L. ., \& Antle, C. E. (1969). Inferences on parameters of the Weibull distribution. Technometrics, 11(3), 445-460.

Thoman, D. R., Bain, L. J., \& Antle, C. E. (1970). Exact confidence intervals for reliability, and tolerance limits in the Weibull distribution. Technometrics, 12(2), 363371.

Weibull, W. (1951). A statistical distribution function of wide applicability. Journal of Applied Mechanics, 18, 293-297. 


\section{Output 2: Quantiles estimates and quantile bounds}

$\$ p$.quantile.estimates

$\begin{array}{rrrr}0.001 \text {-quantile } & 0.005 \text {-quantile } & 0.01 \text {-quantile } & 0.025 \text {-quantile } \\ 259.9 & 593.8 & 848.3 & 1362.5 \\ 0.1 \text {-quantile } & 0.2 \text {-quantile } & 0.3 \text {-quantile } & 0.4 \text {-quantile } \\ 2830.8 & 4159.4 & 5290.4 & 6360.5 \\ 0.6 \text {-quantile } & 0.7 \text {-quantile } & 0.8 \text {-quantile } & 0.9 \text {-quantile } \\ 8582.7 & 9872.7 & 11457.2 & 13767.2 \\ 0.975 \text {-quantile } & 0.99 \text {-quantile } & 0.995 \text {-quantile } & 0.999 \text {-quantile } \\ 17531.0 & 19643.5 & 21107.9 & 24183.6\end{array}$

0.05 -quantile 1957.0

0.5 -quantile 7438.1

0.95 -quantile 15756.3

$\$ p$.quantile.bounds

0.001 -quantile.L

0.001 -quantile.u

0.005 -quantile.L

0.005 -quantile.u

0.01 -quantile.L

0.01 -quantile.u

0.025 -quantile.L

0.025 -quantile.u

0.05 -quantile.L

0.05 -quantile.u

0.1 -quantile.L

0.1 -quantile.u

0.2 -quantile.L

0.2 -quantile. U

0.3 -quantile.L

0.3 -quantile.u

0.4 -quantile.L

0.4 -quantile. U

0.5 -quantile.L

0.5 -quantile.U

0.6 -quantile.L

0.6 -quantile.u

0.7 -quantile.L

0.7 -quantile. $\mathrm{U}$

0.8 -quantile.L

0.8 -quantile.U

0.9 -quantile.L

0.9 -quantile.u

0.95 -quantile. L

$99.5 \%$

1.1

1245.7

8.6

2066.9

20.1

2579.8

62.8

3498.8

159.2

4415.7

398.3

5584.6

1012.6

7417.1

1725.4

8919.8

2548.02848 .2

10616.310130 .4

$3502.4 \quad 3881.1$

$\begin{array}{lll}3892.9 & 10226.8\end{array}$

$\begin{array}{llll}4694.0 & 5022.6 & 5573.8 & 6052.8\end{array}$

$\begin{array}{llllll}6017.1 & 6399.0 & 6876.6 & 7345.8 & 7938.2 & 8628.0\end{array}$

$\begin{array}{lllllll}19271.6 & 17679.9 & 15545.8 & 14181.1 & 12958.0 & 11784.2\end{array}$

$\begin{array}{llllll}7601.3 & 7971.0 & 8465.4 & 8933.5 & 9504.0 & 10244.2\end{array}$

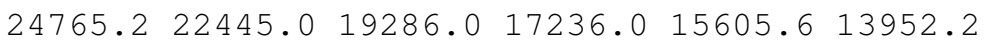

$\begin{array}{lllllll}9674.7 & 10033.7 & 10538.6 & 11031.1 & 11653.0 & 12460.3\end{array}$

$35233.4 \quad 31065.3 \quad 26037.4 \quad 22670.5 \quad 19835.3 \quad 17417.5$

$11203.6 \quad 11584.6 \quad 12145.2 \quad 12660.2 \quad 13365.5 \quad 14311.2$

$46832.9 \quad 40053.3 \quad 32863.127904 .723903 .920703 .0$

$\begin{array}{llllllll}12434.7 & 12833.5 & 13449.7 & 14030.5 & 14781.8 & 15909.1\end{array}$

$\begin{array}{lllllll}59783.1 & 49209.9 & 39397.8 & 33118.7 & 27938.4 & 23773.7\end{array}$

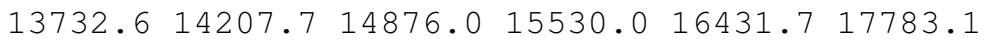

$\begin{array}{llllllll}76425.0 & 61385.4 & 48625.4 & 40067.3 & 33233.8 & 27729.8\end{array}$

$14580.4 \quad 15115.4 \quad 15810.0 \quad 16530.6 \quad 17551.8 \quad 19081.0$

$89690.9 \quad 71480.4 \quad 55033.4 \quad 45187.1 \quad 36918.7 \quad 30505.0$

$16377.7 \quad 16885.9 \quad 17642.5 \quad 18557.1 \quad 19792.4 \quad 21744.7$

$121177.7 \quad 95515.7 \quad 71256.5 \quad 56445.5 \quad 45328.1 \quad 36739.2$ 
Output 3: Tail probability estimates and tail probability bounds

\begin{tabular}{|c|c|c|c|c|c|c|c|}
\hline \multicolumn{6}{|c|}{ Stall.Probability. Estimate } & & \\
\hline 0.36612 & 5977 & .55018 & 0.63402 & 0.70900 & 0.77385 & 0.82821 & 0.87242 \\
\hline $\mathrm{p}(14000)$ & $000)$ & & & & & & \\
\hline 0.90737 & 424 & & & & & & \\
\hline \multicolumn{8}{|c|}{ \$Tail.Probability.Bounds } \\
\hline & $99.5 \%$ & $99 \%$ & $97.5 \%$ & $95 \%$ & $90 \%$ & $80 \%$ & \\
\hline $\mathrm{p}(6000) . \mathrm{L}$ & 0.12173 & 0.13911 & 0.16954 & 0.19782 & 0.23300 & 0.28311 & \\
\hline $\mathrm{p}(6000) . \mathrm{U}$ & 0.69856 & 0.67056 & 0.63572 & 0.59592 & 0.54776 & 0.49023 & \\
\hline $\mathrm{p}(7000) . \mathrm{L}$ & 0.17411 & 0.20130 & 0.23647 & 0.26985 & 0.31017 & 0.36523 & \\
\hline $\mathrm{p}(7000) . \mathrm{U}$ & 0.76280 & 0.73981 & 0.70837 & 0.67426 & 0.62988 & 0.57670 & \\
\hline $\mathrm{p}(8000) . \mathrm{L}$ & 0.23898 & 0.26838 & 0.30397 & 0.34488 & 0.38942 & 0.44487 & \\
\hline $\mathrm{p}(8000) . \mathrm{U}$ & 0.82187 & 0.80141 & 0.77310 & 0.74260 & 0.70414 & 0.65435 & \\
\hline$p(9000) . L$ & 0.30561 & 0.33149 & 0.37276 & 0.41748 & 0.46448 & 0.52203 & \\
\hline$p(9000) . U$ & 0.87042 & 0.85462 & 0.82993 & 0.80361 & 0.77045 & 0.72545 & \\
\hline $\mathrm{p}(1000000) . \mathrm{L}$ & 0.36871 & 0.39257 & 0.44219 & 0.48549 & 0.53589 & 0.59276 & \\
\hline $\mathrm{p}\left(\begin{array}{lllll}1 & 0 & 0 & 0 & 0\end{array}\right) . \mathrm{U}$ & 0.91227 & 0.89889 & 0.87805 & 0.85624 & 0.82667 & 0.78641 & \\
\hline $\mathrm{p}(11000) . \mathrm{L}$ & 0.41612 & 0.45097 & 0.50030 & 0.54631 & 0.59749 & 0.65671 & \\
\hline $\mathrm{p}(11000) . \mathrm{U}$ & 0.94491 & 0.93318 & 0.91728 & 0.89891 & 0.87425 & 0.83973 & \\
\hline $\mathrm{p}(12000) . \mathrm{L}$ & 0.46351 & 0.50388 & 0.55531 & 0.60133 & 0.65374 & 0.71215 & \\
\hline $\mathrm{p}(12000) . \mathrm{U}$ & 0.96669 & 0.95936 & 0.94650 & 0.93210 & 0.91231 & 0.88377 & \\
\hline $\mathrm{p}(13000) . \mathrm{L}$ & 0.50876 & 0.54776 & 0.60262 & 0.65055 & 0.70218 & 0.76148 & \\
\hline $\mathrm{p}(13000) . \mathrm{U}$ & 0.98278 & 0.97742 & 0.96794 & 0.95756 & 0.94149 & 0.91745 & \\
\hline $\mathrm{p}(14000) . \mathrm{L}$ & 0.54668 & 0.58696 & 0.64619 & 0.69359 & 0.74451 & 0.80178 & \\
\hline $\mathrm{p}(14000) . \mathrm{U}$ & 0.99201 & 0.98837 & 0.98205 & 0.97459 & 0.96267 & 0.94321 & \\
\hline $\mathrm{p}(15000) . \mathrm{L}$ & 0.58089 & 0.62534 & 0.68389 & 0.73194 & 0.78068 & 0.83590 & \\
\hline $\mathrm{p}(15000) . \mathrm{U}$ & 0.99653 & 0.99449 & 0.99092 & 0.98596 & 0.97764 & 0.96268 & \\
\hline
\end{tabular}

\section{Citation}

Scholz, F. W. (2015) Inference for the Weibull Distribution: A tutorial. The Quantitative Methods for Psychology, 11(3), $148-173$.

Copyright () 2015 Scholz. This is an open-access article distributed under the terms of the Creative Commons Attribution License (CC BY). The use, distribution or reproduction in other forums is permitted, provided the original author(s) or licensor are credited and that the original publication in this journal is cited, in accordance with accepted academic practice. No use, distribution or reproduction is permitted which does not comply with these terms.

Received: 19/08/2015 Accepted: 25/08/2015

Figures and one listing follows on next page 
Listing 1 ॥ Computation of the maximum likelihood estimates of alpha and beta for complete or type II censored samples assumed to come from a 2-parameter Weibull distribution

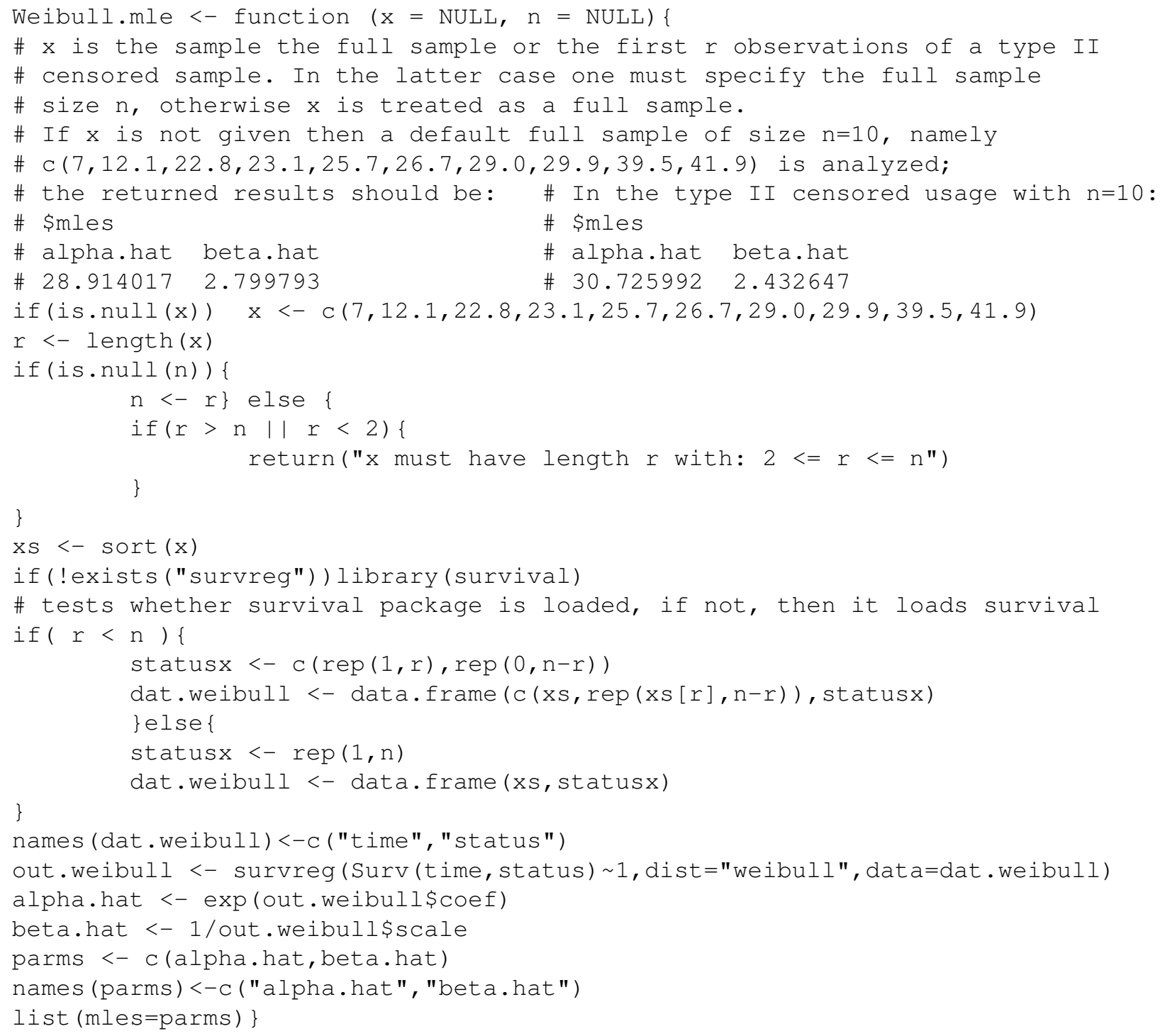


Figure 7 Interpolation \& Extrapolation for $\sqrt{n} \times D$

(a)

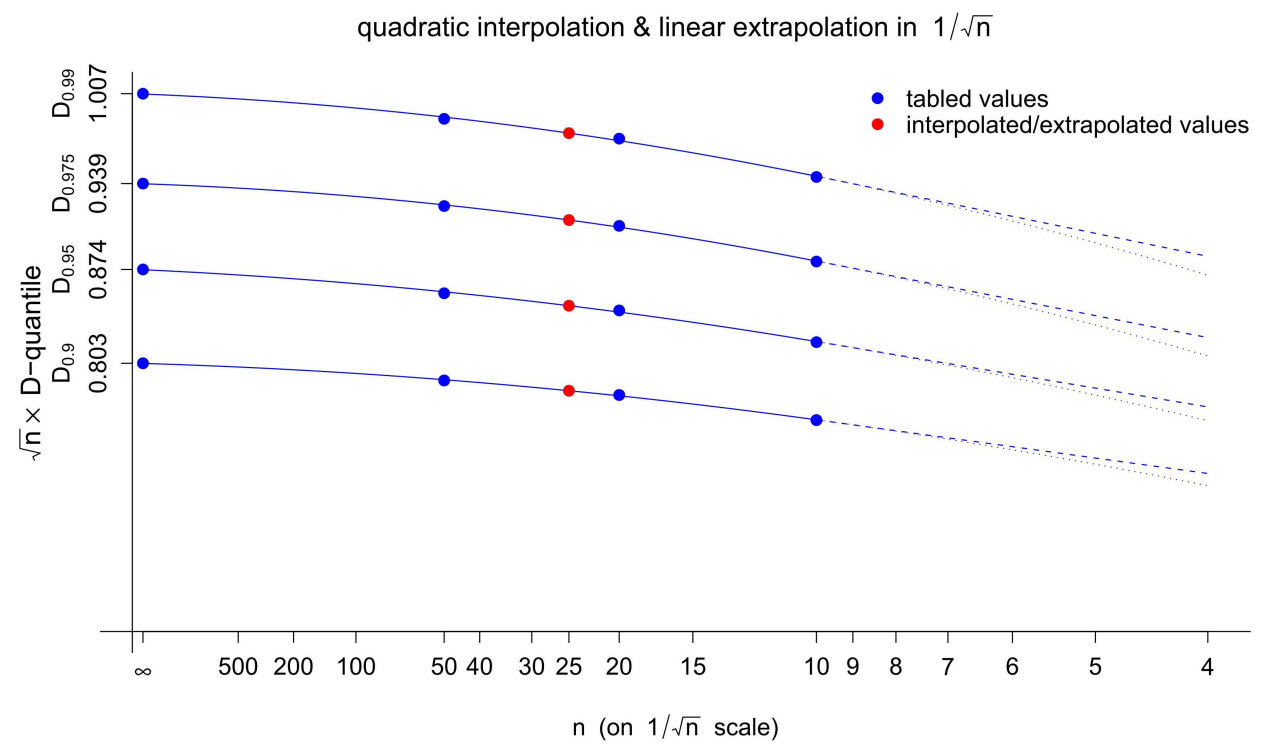

(b)

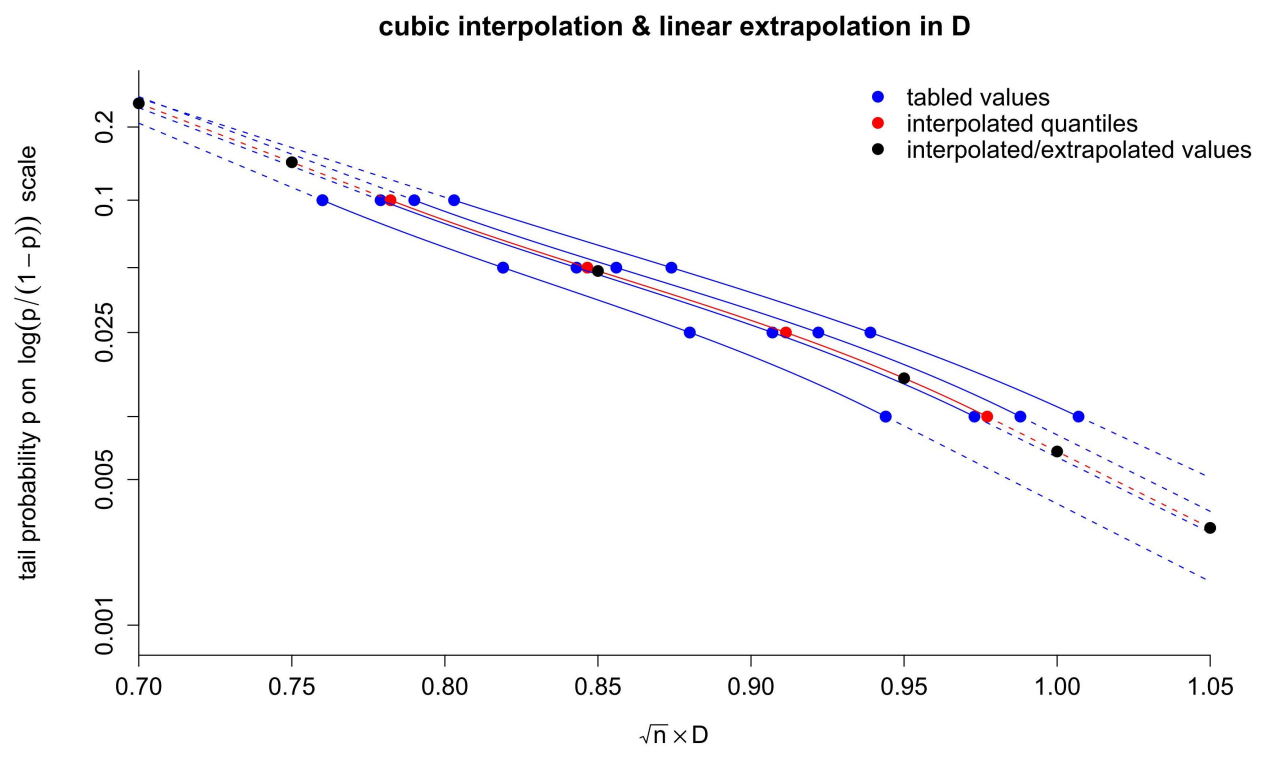


Figure 8 - Abnormal Behavior of Tabulated Confidence Quantiles

(a)

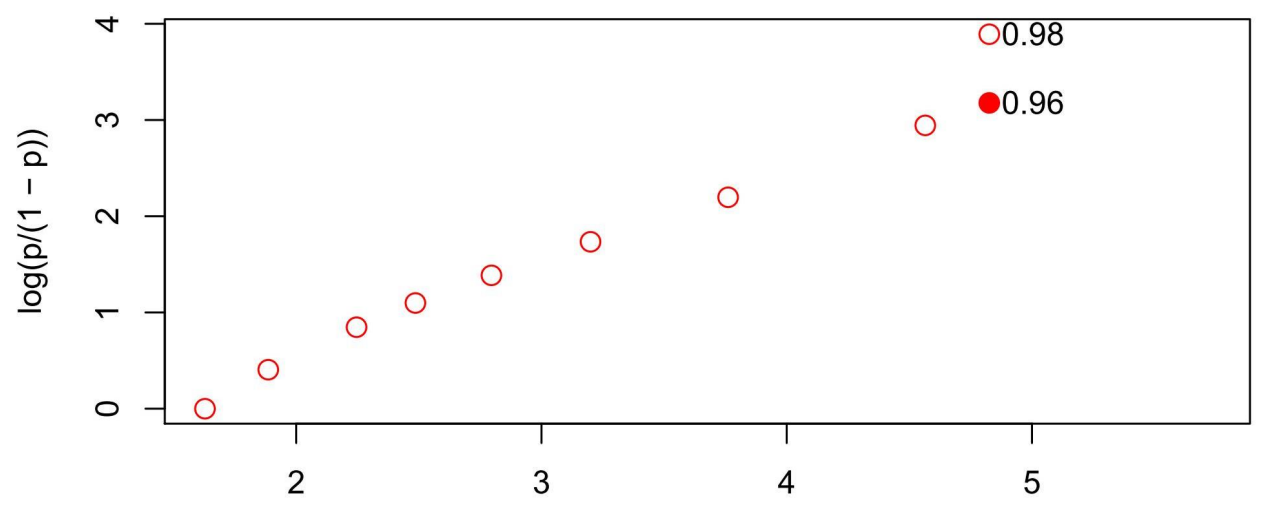

Bain's tabled quantiles for $n=40, \gamma=0.9$

(b)

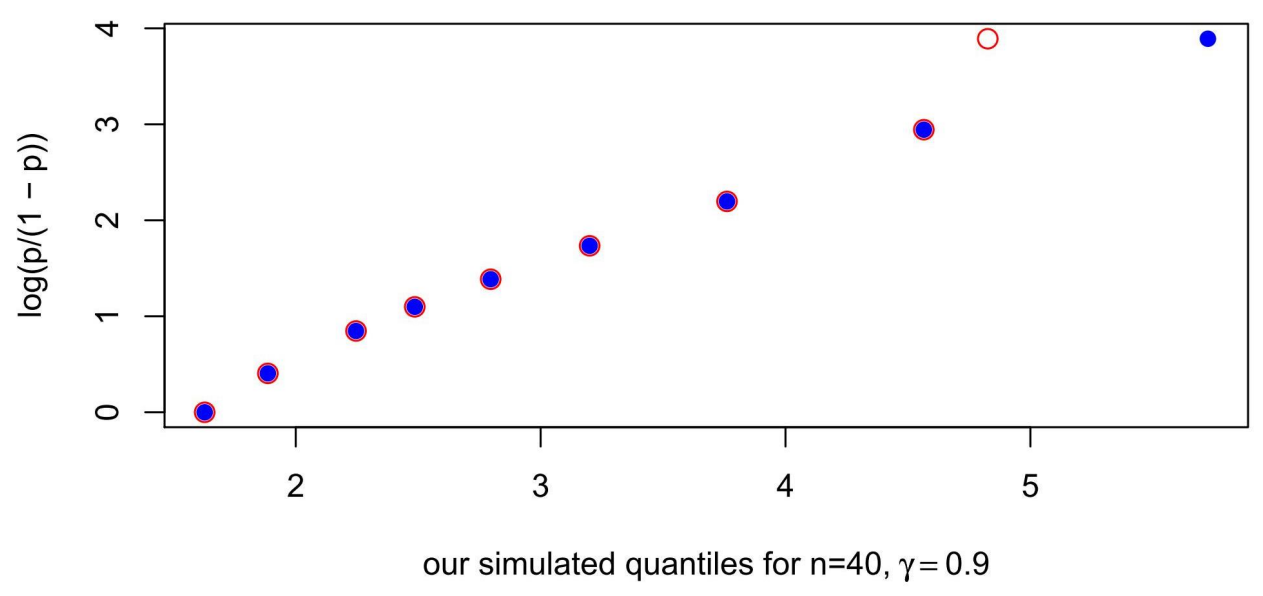

Figure 9 - Timings for Weibul lP i vots for Various $n$

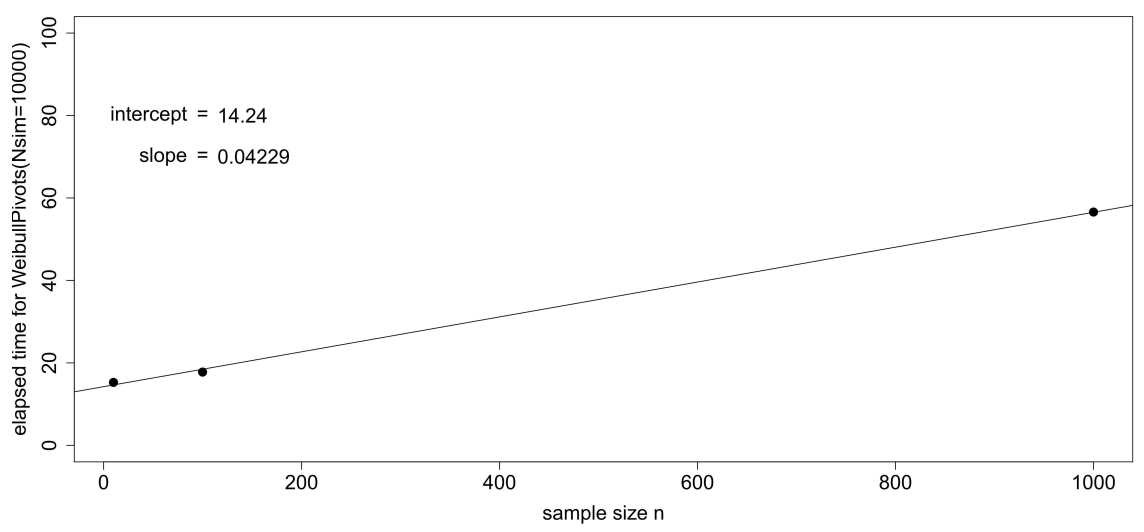


Figure 10 $\approx$ Pivot Distributions of $\hat{u} / \hat{b}$ and $\hat{b}$

(a)

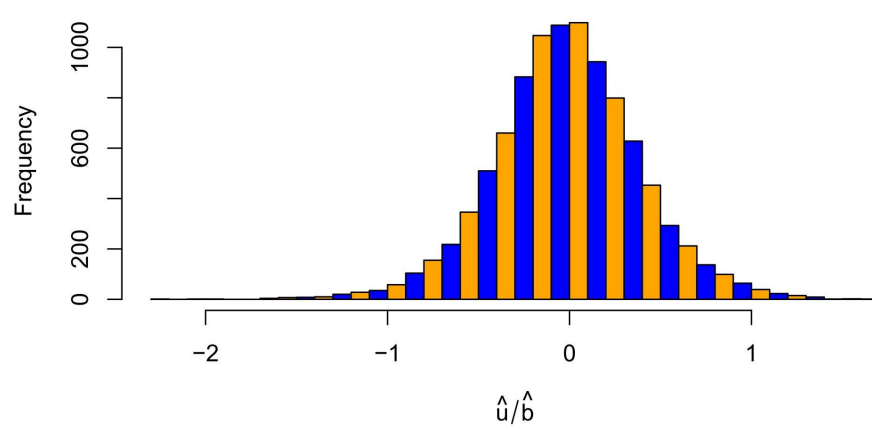

(b)

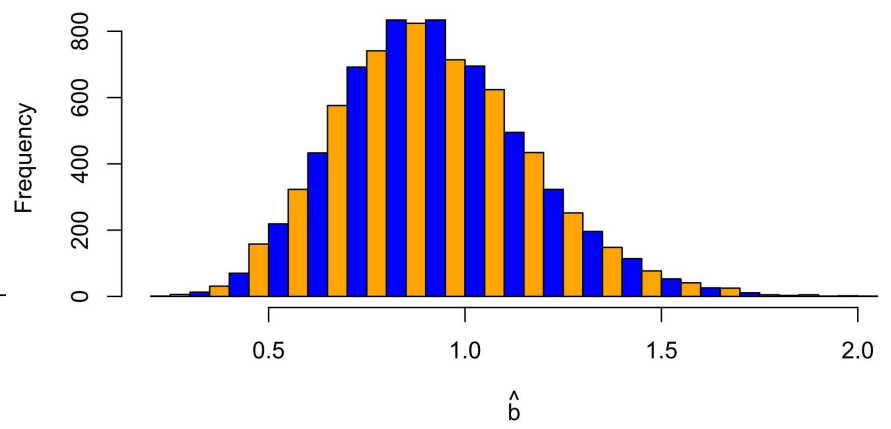

Figure 11 - Weibull Plot Corresponding to Previous Output

Weibull Plot

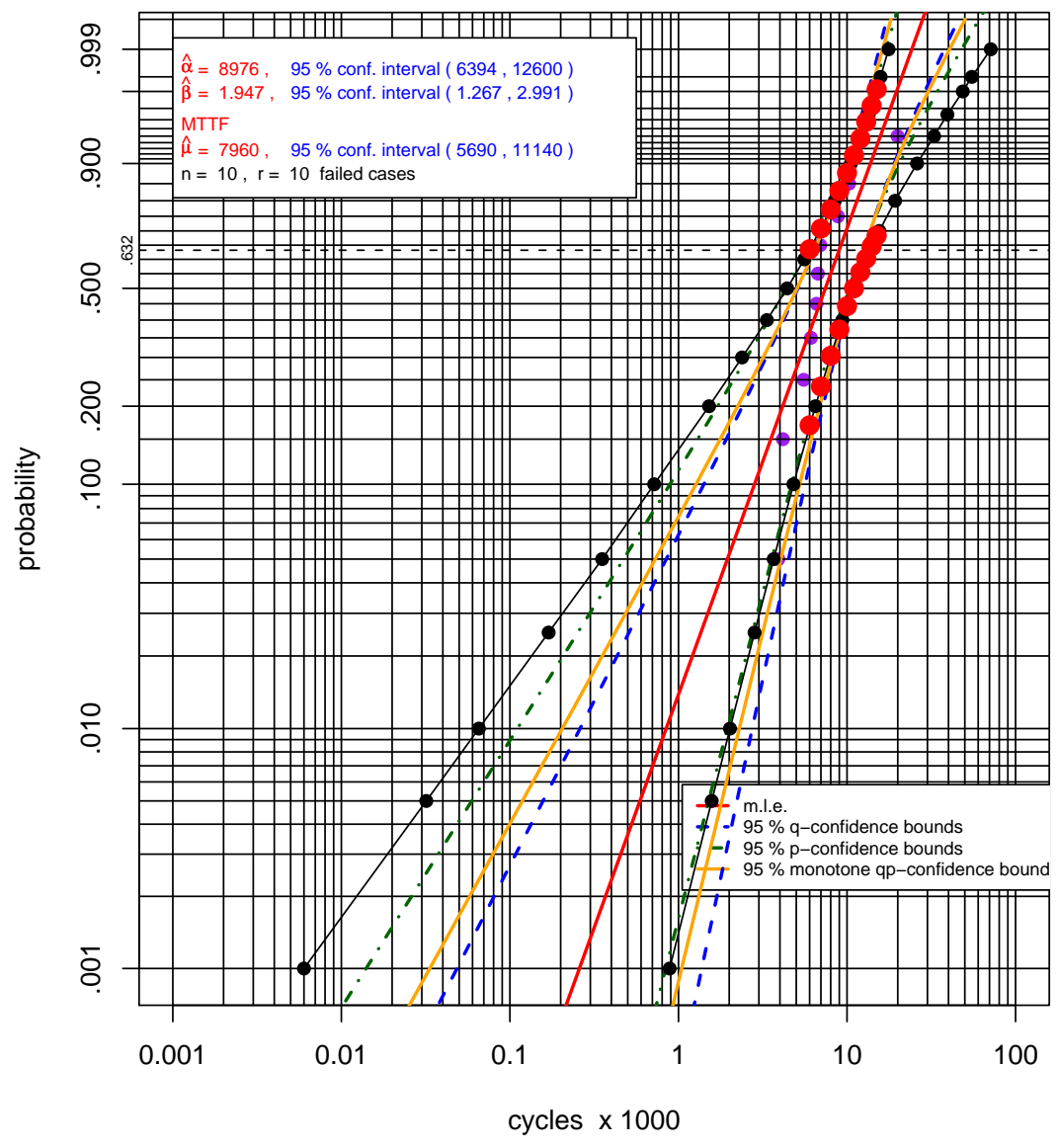


Figure 12 - Weibull Plot for Weibull Sample of Size $n=2$

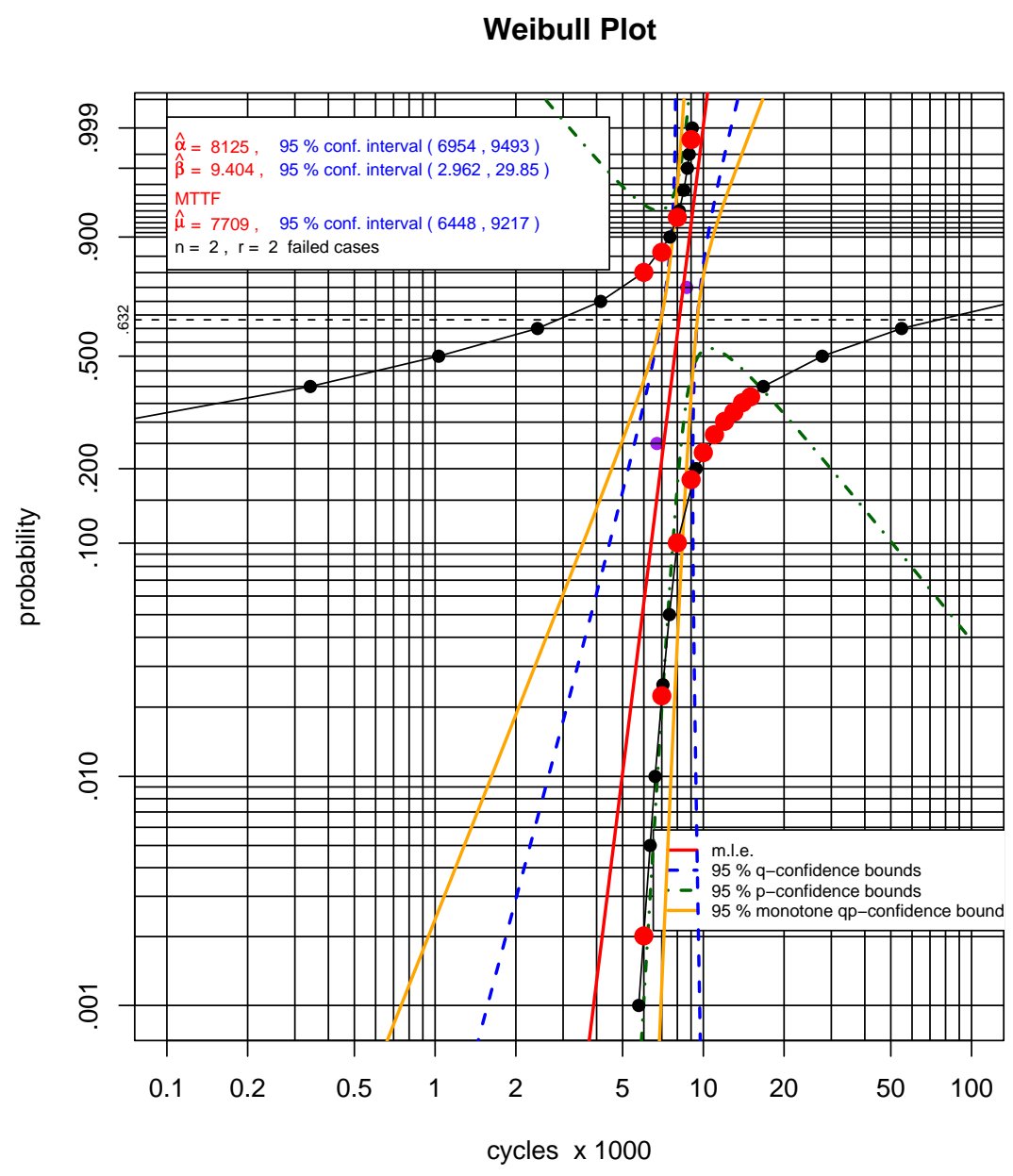

